\title{
Ring Opening Metathesis/Oxy-Cope Rearrangement: A General Strategy for the Synthesis of Bicyclic Medium Ring-Containing Compounds
}

\author{
Brian H. White and Marc L. Snapper* \\ Eugene F. Merkert Chemistry Center, Boston College, Chestnut Hill, Massachusetts, \\ 02467
}

\section{Supporting Information}

General: Starting materials and reagents were purchased from commercial suppliers and were used without further purification with the following exceptions: tetrahydrofuran, diethyl ether, dichloromethane and benzene used in reactions were dried on alumina columns using a solvent dispensing system; ${ }^{1} 18$-crown-6 was recrystallized from acetonitrile and dried in vacuo for one day prior to use; hexanes and diethyl ether used in chromatography were distilled prior to use; 4,4,5,5-tetramethyl-2-vinyl-[1,3,2]dioxaborolane was made following literature precedent ${ }^{2}$ and stored in frozen benzene. All reactions were performed in flamedried glassware under an inert atmosphere of nitrogen unless otherwise noted.

Infrared (FTIR) spectra were recorded on a Nicolet 210 FT-infrared spectrometer, and reported in wavenumbers $\left(\mathrm{cm}^{-1}\right)$. Bands are characterized as broad (b), strong (s), medium (m) or weak (w). Proton nuclear magnetic resonance ( ${ }^{1} \mathbf{H}$ NMR) spectra were recorded on either a Varian Gemini-400 instrument $(400 \mathrm{MHz})$, or a Varian Gemini-500 instrument (500 MHz). Chemical shifts are reported with the solvent as the reference $\left(\mathrm{CDCl}_{3}: \square 7.27 \mathrm{ppm}, \mathrm{C}_{6} \mathrm{D}_{6}: \square 7.16 \mathrm{ppm}\right)$. Data is reported as follows: chemical shift, multiplicity $(\mathrm{s}=$ singlet, $\mathrm{d}=$ doublet, $\mathrm{t}=$ triplet, $\mathrm{q}=$ quartet, $\mathrm{br}=$ broad, $\mathrm{m}=$ multiplet $)$, coupling constants $(\mathrm{Hz})$, and integration. Carbon nuclear magnetic resonance $\left({ }^{13} \mathbf{C}\right.$ NMR) spectra were recorded on either a Varian Gemini-400 instrument (100 MHz) or a Varian Gemini-500 instrument (125 MHz) with complete decoupling. Chemical shifts are reported with the solvent as reference $\left(\mathrm{CDCl}_{3}: \square 77.23 \mathrm{ppm}, \mathrm{C}_{6} \mathrm{D}_{6} \square 128.02\right.$ ppm). Elemental analyses (Anal.) were performed by Robinson Microlit Laboratories Inc, Madison, NJ and are reported in percent abundance. High resolution mass spectral analyses (HRMS) were performed by Mass Spectrometry Facility, Boston College.

X-ray data was collected using a Bruker APEX CCD (charged coupled deviced) based diffractometer equipped with an LT-2 low temperature apparatus operating at $193 \mathrm{~K}$. A suitable crystal was chosen and mounted on a glass fiber using grease. Data was measured using omega scans of $0.3^{\circ}$ per frame for either 30 (XV, XVI) or 45 (all others) seconds, such that a hemisphere was collected. A total of 1305 frames were collected with a maximum resolution of $0.90 \AA$. Cell parameters were retrieved using

\footnotetext{
${ }^{1}$ Pangborn, A. B.; Giardello, M. A.; Grubbs, R. H.; Rosen, R. K.; Timmers, F. J. Organometallics 1996, 15, 1518.

${ }^{2}$ Wallace, R. H.; Zong, K. K. Tetrahedron Lett. 1992, 33, 6941.
} 
$\mathrm{SMART}^{3}$ software and refined using SAINT on all observed reflections. Data reduction was performed using the SAINT software ${ }^{4}$, which corrects for Lp and decay. Absorption corrections were applied using SADABS supplied by George Sheldrick. The structures ware solved by the direct method using the SHELXS- $97^{5}$ program and refined by least squares method on $\mathrm{F}^{2}$, SHELXL-97 $7^{6}$, incorporated in SHELXTL-PC V $6.10^{7}$.

All non-hydrogen atoms are refined anisotropically. Hydrogens were calculated by geometrical methods and refined as a riding model. The crystals used for the diffraction study showed no decomposition during data collection. All drawings are done at 30\% ellipsoids. The structures were solved by Jarred T. Blank at Boston College.

\section{Experimental Procedures:}

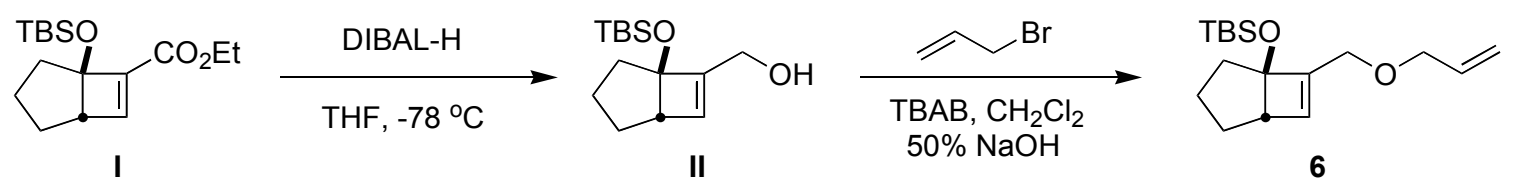

A solution of cyclobutene $\mathbf{I}(6.55 \mathrm{~g}, 22.1 \mathrm{mmol})$ in THF $(50 \mathrm{~mL})$ was cooled to $-78{ }^{\circ} \mathrm{C}$, and DIBAL-H $(9.8$ $\mathrm{mL}, 54.8 \mathrm{mmol}$ ) was added dropwise to the solution. After stirring at $-78{ }^{\circ} \mathrm{C}$ for $4 \mathrm{~h}$, the reaction was poured slowly into a mixture of ethyl acetate $(100 \mathrm{~mL})$ and saturated aqueous sodium potassium tartrate $(500 \mathrm{~mL})$. The mixture was stirred until both layers turned clear, and additional ethyl acetate $(400 \mathrm{~mL})$ was added. The organic extracts were dried with $\mathrm{MgSO}_{4}$ and concentrated in vacuo. Purification of the remaining oil by chromatography on silica gel (93:7 pentane:ether) gave alcohol II (4.56 g, 17.9 mmol, $81 \%$ yield) as a colorless oil.

Compound II: FTIR (NaCl, thin film) 3257 (b), 2948 (s), 2854 (s), 1463 (w), 1249 (m), 1174 (m), 1111 (m), 1060 (m), 897 (m), 834 (m), 777 (m) cm ${ }^{-1} .{ }^{1} \mathbf{H}$ NMR (400 MHz, $\left.\mathrm{CDCl}_{3}\right)$ : $\square 5.75$ (s, 1H), 4.20 (d, J= $14.8 \mathrm{~Hz}, 1 \mathrm{H}), 4.11(\mathrm{dd}, J=14.8,5.2 \mathrm{~Hz}, 1 \mathrm{H}) 2.86(\mathrm{br} \mathrm{s}, 1 \mathrm{H}), 1.92-1.84(\mathrm{~m}, 2 \mathrm{H}), 1.70(\mathrm{~m}, 1 \mathrm{H}), 1.46-1.34$ (m, 3H), 0.87 (s, 9H), 0.10 (s, 6H). ${ }^{13} \mathbf{C}$ NMR (100 MHz, $\mathrm{CDCl}_{3}$ ): $\mathrm{1}$ 149.5, 127.1, 88.4, 58.8, 52.4, 33.8, 26.0, 25.9, 23.7, 18.3, -2.6, -2.8. Anal. Calcd. for $\mathrm{C}_{14} \mathrm{H}_{26} \mathrm{O}_{2} \mathrm{Si}$ : C, 66.09; H, 10.30. Found: $\mathrm{C}, 65.80 ; \mathrm{H}$, 10.16

\footnotetext{
${ }^{3}$ SMART V5.626 (NT) Software for the CCD Detector Systems; Bruker Analytical X-ray Systems, Madison, WI (2001)

${ }^{4}$ SAINT V 5.01 (NT) Software for the CCD Detector Systems; Bruker Analytical X-ray Systems, Madison, WI (2001)

${ }^{5}$ Sheldrick, G. M. SHELXS-90, Program for the Solution of Crystal Structure, University of Göttingen, Germany, 1990.

${ }^{6}$ Sheldrick, G. M. SHELXL-97, Program for the Refinement of Crystal Structure, University of Göttingen, Germany, 1997.

${ }^{7}$ SHELXTL 6.0 (PC-Version), Program Library for Structure Solution and Molecular Graphics; Bruker Analytical Xray Systems, Madison, WI (1998)
} 
A flask under ambient conditions was charged with alcohol II (1.02 g, $4.01 \mathrm{mmol})$, and $\mathrm{CH}_{2} \mathrm{Cl}_{2}(10 \mathrm{~mL})$ was added. Allyl bromide $(3.46 \mathrm{~mL}, 40.0 \mathrm{mmol})$ was added, followed by tetrabutylammonium bromide (322 mg, $1.00 \mathrm{mmol}$ ) and a $50 \%$ solution of aqueous $\mathrm{NaOH}(5 \mathrm{~mL})$. After stirring for $24 \mathrm{~h}$, the reaction mixture was poured into $1 \mathrm{~N} \mathrm{HCl}(100 \mathrm{~mL})$, and extracted with ethyl acetate $(100 \mathrm{~mL})$. The organic layer was dried with $\mathrm{MgSO}_{4}$ and concentrated in vacuo. Purification of the remaining oil by chromatography on silica gel (98:2 hexanes:ether) gave ether 6 (937 mg, $3.18 \mathrm{mmol}, 80 \%$ yield) as a colorless oil.

Compound 6: FTIR (NaCl, thin film) 2948 (s), 2854 (s), 1463 (w), 1318 (w), 1255 (m), 1180 (m), 1117 (m), 897 (m), 840 (m), 777 (m) cm ${ }^{-1} .{ }^{1} \mathbf{H}$ NMR (400 MHz, $\mathrm{CDCl}_{3}$ ): $\square 5.92$ (ddt, $\left.J=16.4,10.8,5.6 \mathrm{~Hz}, 1 \mathrm{H}\right)$, 5.79 (s, 1H), 5.28 (dd, $J=16.4,1.6 \mathrm{~Hz}, 1 \mathrm{H}), 5.18$ (dd, $J=10.4,1.6 \mathrm{~Hz}, 1 \mathrm{H}), 4.02-3.95$ (m, 4H), 2.83 (br s, $1 \mathrm{H}), 1.85(\mathrm{dd}, J=12.0,5.8 \mathrm{~Hz}, 1 \mathrm{H}), 1.69-1.67(\mathrm{~m}, 1 \mathrm{H}), 1.47-1.29(\mathrm{~m}, 4 \mathrm{H}), 0.87$ (s, 9H), 0.09 (s, 3H), 0.08 (s, 3H). ${ }^{13} \mathbf{C}$ NMR (100 MHz, $\left.\mathrm{CDCl}_{3}\right):$ : 147.9, 134.8, 130.5, 116.9, 87.9, 71.7, 64.9, 52.8, 54.2, 26.0, 25.9, 23.8, 18.4, -2.6, -2.9. Anal. Calcd. for $\mathrm{C}_{17} \mathrm{H}_{30} \mathrm{O}_{2} \mathrm{Si}$ : C, 69.33; H, 10.27. Found: C, 69.18; H, 10.14 .

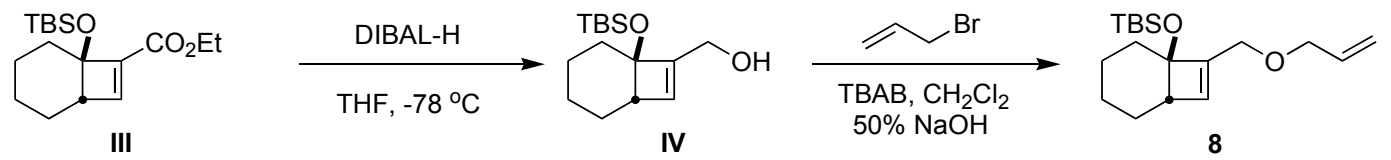

A solution of ester III $(5.14 \mathrm{~g}, 16.5 \mathrm{mmol})$ in THF $(50 \mathrm{~mL})$ was cooled to $-78{ }^{\circ} \mathrm{C}$. DIBAL-H $(8.8 \mathrm{~mL}, 50.0$ mmol) was added dropwise. After stirring for $3 \mathrm{~h}$ at $-78{ }^{\circ} \mathrm{C}$, the reaction was slowly poured into a mixture of ethyl acetate $(100 \mathrm{~mL})$ and saturated aqueous sodium potassium tartrate $(500 \mathrm{~mL})$. The mixture was stirred until both layers turned clear, and additional ethyl acetate $(400 \mathrm{~mL})$ was added. The organic extracts were dried with $\mathrm{MgSO}_{4}$, concentrated in vacuo, and the remaining oil purified by chromatography on silica gel (93:7 pentane:ether) to yield alcohol IV (3.74 g, $13.9 \mathrm{mmol}, 84 \%)$ as a colorless oil.

Compound IV: FTIR (NaCl, thin film): 3330 (b), 2929 (s), 2856 (s), 1471 (m), 1254 (s), 1126 (m), 878 (m), $835(\mathrm{~s}), 773(\mathrm{~m}) \mathrm{cm}^{-1} .{ }^{1} \mathbf{H}$ NMR (400 MHz, $\left.\mathrm{CDCl}_{3}\right): \square 5.97(\mathrm{~s}, 1 \mathrm{H}), 4.20(\mathrm{~d}, J=14.4 \mathrm{~Hz}, 1 \mathrm{H}), 4.12$ (dd, $J=14.8,3.6 \mathrm{~Hz}, 1 \mathrm{H}), 2.71$ (br s, 1H), 2.01 (br s, 1H), 1.82-1.70 (m, 3H), 1.56-1.43 (m, 4H), 0.86 (s, 9H), 0.10 (s, 3H), 0.09 (s, 3H). ${ }^{13} \mathbf{C}$ NMR (100 MHz, $\mathrm{CDCl}_{3}$ ): $\square$ 151.2, 130.5, 79.2, 59.2, 49.2, 32.5, 26.0, 23.5, 18.3, 17.8, -2.4, -2.7. Anal. Calcd. for $\mathrm{C}_{15} \mathrm{H}_{28} \mathrm{O}_{2} \mathrm{Si}$ : C, 67.11; H, 10.51. Found: C, 66.83; H, 10.25.

A flask under ambient conditions was charged with alcohol IV (1.17 g, $4.36 \mathrm{mmol})$, and $\mathrm{CH}_{2} \mathrm{Cl}_{2}(10 \mathrm{~mL})$ added. Allyl bromide $(1.88 \mathrm{~mL}, 21.8 \mathrm{mmol})$ was added, followed by tetrabutylammonium bromide $(250$ $\mathrm{mg}, 0.775 \mathrm{mmol})$ and a $50 \%$ solution of aqueous $\mathrm{NaOH}(2.5 \mathrm{~mL})$. After stirring for $24 \mathrm{~h}$, the reaction mixture was poured into $1 \mathrm{~N} \mathrm{HCl}(100 \mathrm{~mL})$, and extracted with ethyl acetate $(100 \mathrm{~mL})$. The organic layer was dried with $\mathrm{MgSO}_{4}$ and concentrated in vacuo. Purification of the remaining oil by chromatography on silica gel (98:2 hexanes:ether) gave ether $8(1.05 \mathrm{mg}, 3.40 \mathrm{mmol}, 78 \%$ yield $)$ as a colorless oil. 
Compound 8: FTIR (NaCl, thin film): 2930 (s), 2854 (s), 1463 (w), 1362 (w), 1255 (m), 1136 (m), 1105 (s), 991 (w), 878 (m), 840 (s), 777 (m) cm ${ }^{-1} .{ }^{1} \mathbf{H}$ NMR (400 MHz, CDCl $)$ : $\square 6.01$ (s, 1H), 5.93 (ddt, $J=$ $17.2,11.2,5.6 \mathrm{~Hz}, 1 \mathrm{H}), 5.29$ (dd, $J=17.2,1.6 \mathrm{~Hz}, 1 \mathrm{H}), 5.18(\mathrm{dd}, J=9.2,1.6 \mathrm{~Hz}, 1 \mathrm{H}), 4.07-3.96(\mathrm{~m}, 4 \mathrm{H})$, 2.68 (br s, 1H), 1.76-1.70 (m, 3H), 1.53-1.46 (m, 5H), 0.86 (s, 9H), 0.09 (s, 3H), 0.07 (s, 3H). ${ }^{13} \mathbf{C}$ NMR (100 MHz, $\left.\mathrm{CDCl}_{3}\right): \square$ 150.2, 134.9, 131.6, 116.9, 78.6, 71.8, 65.2 , 49.6, 32.7, 26.1, 23.6, 18.4, 18.2, 18.0, -2.4, -2.6. Anal. Calcd. for $\mathrm{C}_{18} \mathrm{H}_{32} \mathrm{O}_{2} \mathrm{Si}$ : C, 70.07; H, 10.45. Found: C, 69.85; H, 10.32 .
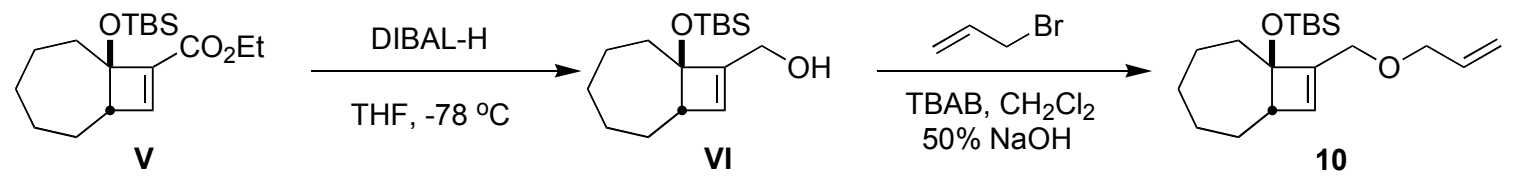

A solution of ester $\mathbf{V}(1.48 \mathrm{~g}, 4.56 \mathrm{mmol})$ in THF $(15 \mathrm{~mL})$ was cooled to $-78^{\circ} \mathrm{C}$. DIBAL-H $(1.87 \mathrm{~mL}, 10.5$ mmol) was added dropwise. After stirring for $3 \mathrm{~h}$ at $-78{ }^{\circ} \mathrm{C}$, the reaction was slowly poured into a mixture of ethyl acetate $(50 \mathrm{~mL})$ and saturated aqueous sodium potassium tartrate $(250 \mathrm{~mL})$. The mixture was stirred until both layers turned clear, and additional ethyl acetate $(200 \mathrm{~mL})$ was added. The organic extracts were dried with $\mathrm{MgSO}_{4}$, concentrated in vacuo, and the remaining oil purified by chromatography on silica gel (93:7 pentane:ether) to yield alcohol VI (1.04 g, $3.68 \mathrm{mmol}, 81 \%)$ as a colorless oil.

Compound VI: FTIR (NaCl, thin film): 3323 (b), 2953 (s), 2854 (s), 1460 (m), 1359 (w), 1252 (m), 1192 (m), 1103 (s), 1073 (s), 834 (s), 773 (m) cm ${ }^{-1} .{ }^{1} \mathbf{H}$ NMR (400 MHz, CDCl $)$ : $\square 6.01$ (s, 1H), 4.18 (br d, $J=$ $14.8 \mathrm{~Hz}, 1 \mathrm{H}), 4.12$ (br d, $J=14.8 \mathrm{~Hz}, 1 \mathrm{H}), 2.81$ (br d, $J=6.4 \mathrm{~Hz}, 1 \mathrm{H}), 1.88-1.60$ (m, $6 \mathrm{H}), 1.48-1.38$ (m, 4H), 0.88 (s, 9H), 0.13 (s, 3H), 0.08 (s, 3H). ${ }^{13} \mathbf{C}$ NMR (100 MHz, $\left.\mathrm{CDCl}_{3}\right): \square 152.0,131.2,83.9,58.9,55.5$, 37.1, 32.6, 30.7, 27.3, 26.1, 24.4, 18.5, -2.2, -2.8. Anal. Calcd. for $\mathrm{C}_{16} \mathrm{H}_{30} \mathrm{O}_{2} \mathrm{Si}$ : C, 68.03; H, 10.70. Found: C, 67.99; H, 10.55 .

A flask under ambient conditions was charged with alcohol VI (638 mg, $2.26 \mathrm{mmol})$, and $\mathrm{CH}_{2} \mathrm{Cl}_{2}$ (5 mL) added. Allyl bromide $(0.98 \mathrm{~mL}, 11 \mathrm{mmol})$ was added, followed by TBAB $(100 \mathrm{mg}, 0.310 \mathrm{mmol})$ and a $50 \%$ solution of aqueous $\mathrm{NaOH}(1.0 \mathrm{~mL})$. After stirring for $24 \mathrm{~h}$, the reaction mixture was poured into $1 \mathrm{~N}$ $\mathrm{HCl}(50 \mathrm{~mL})$, and extracted with ethyl acetate $(50 \mathrm{~mL})$. The organic layer was dried with $\mathrm{MgSO}_{4}$ and concentrated in vacuo. Purification of the remaining oil by chromatography on silica gel (98:2 hexanes:ether) gave ether $\mathbf{1 0}$ (700 mg, $2.17 \mathrm{mmol}, 96 \%$ yield) as a colorless oil.

Compound 10: FTIR (NaCl, thin film): 2930 (s), 2860 (s), 1463 (w), 1362 (w), 1255 (m), 1195 (m), 111 (s), 1075 (s), 993 (m), 922 (m), 840 (s), 777 (m) cm ${ }^{-1} .{ }^{1} \mathbf{H}$ NMR (400 MHz, CDCl $)$ : $\square 6.02$ (br s, 1H), 5.91 (ddt, $J=16.0,10.4,5.6 \mathrm{~Hz}, 1 \mathrm{H}), 5.29$ (dq, $J=17.0,1.6 \mathrm{~Hz}, 1 \mathrm{H}$ ), 5.19 (dq, $J=10.4,1.6 \mathrm{~Hz}, 1 \mathrm{H}$ ), 4.06-3.94 (m, 4H), 2.75 (br d, $J=6.0 \mathrm{~Hz}, 1 \mathrm{H}), 1.87-1.77$ (m, 2H), 1.70-1.62 (m, 4H), 1.53-1.48 (m, 1H), 1.36-1.32 (m, 3H), 0.88 (s, 9H), 0.12 (s, 3H), 0.06 (s, 3H). ${ }^{13} \mathbf{C}$ NMR (100 MHz, $\left.\mathrm{CDCl}_{3}\right): \square$ 150.0, 134.8, 132.5, 
117.0, 83.5, 71.8, 65.1, 56.1, 37.0, 32.5, 31.1, 27.6, 26.1, 24.3, 18.6, -2.3, -2.8. Anal. Calcd. for $\mathrm{C}_{19} \mathrm{H}_{34} \mathrm{O}_{2} \mathrm{Si}: \mathrm{C}, 70.75 ; \mathrm{H}, 10.62$. Found: $\mathrm{C}, 70.64 ; \mathrm{H}, 10.72$.

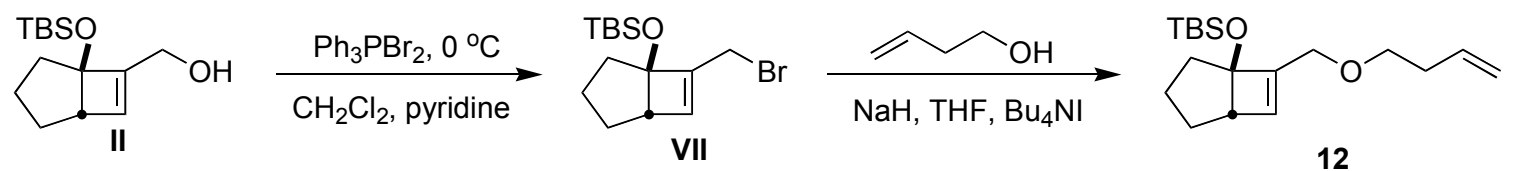

A flask was charged with $\mathrm{Ph}_{3} \mathrm{PBr}_{2}(1.75 \mathrm{~g}, 4.14 \mathrm{mmol})$, dissolved in $\mathrm{CH}_{2} \mathrm{Cl}_{2}(50 \mathrm{~mL})$, and cooled to $0{ }^{0} \mathrm{C}$. A second solution of alcohol II $(947 \mathrm{mg}, 3.72 \mathrm{mmol})$ and pyridine $(0.42 \mathrm{~mL}, 5.2 \mathrm{mmol})$ in $\mathrm{CH}_{2} \mathrm{Cl}_{2}(50 \mathrm{~mL})$ was cannulated into the first solution, and stirred for $1 \mathrm{~h}$. The reaction mixture was washed with saturated $\mathrm{NaHCO}_{3}(50 \mathrm{~mL})$; the organic layer was dried with $\mathrm{MgSO}_{4}$ and concentrated in vacuo. Purification of the remaining oil by chromatography on silica gel (100\% hexanes) gave bromide VII (1.04 g, $3.26 \mathrm{mmol}$, 88\% yield) as a faint yellow oil.

Compound VII: FTIR (NaCl, thin film): 3037 (w), 2955 (s), 2854 (s), 1463 (m), 1325 (m), 1256 (m), 1211 (m), 1174 (s), 1111 (s), 903 (s), 840 (s), 777 (s), 683 (m) cm ${ }^{-1} .{ }^{1} \mathbf{H}$ NMR (400 MHz, CDCl $)$ : $\square 5.92$ (s, 1H), 3.85 (s, 2H), $2.82(\mathrm{br} \mathrm{d}, J=3.6 \mathrm{~Hz}, 1 \mathrm{H}), 2.04(\mathrm{dd}, J=12.0,4.8 \mathrm{~Hz}, 1 \mathrm{H}), 1.71-1.69(\mathrm{~m}, 1 \mathrm{H})$, 1.51-1.44 (m, 2H), 1.38-1.27 (m, 2H), 0.89 (s, 9H), 0.10 (s, 3H), 0.09 (s, 3H). ${ }^{13} \mathbf{C}$ NMR (100 MHz, $\left.\mathrm{CDCl}_{3}\right)$ : $\square$ 145.8, 132.6, 88.5, 52.5, 34.2, 26.1, 26.0, 24.2, 23.6, 18.4, -2.6, -2.7. Anal. Calcd. for $\mathrm{C}_{14} \mathrm{H}_{25} \mathrm{BrOSi}$ : C, 52.99; H, 7.94. Found: C, 53.23; H, 7.78.

A flask was charged with $\mathrm{NaH}(185 \mathrm{mg}, 60 \%$ suspension in mineral oil, $4.62 \mathrm{mmol})$ and washed with hexanes $(2 \times 5 \mathrm{~mL})$. Tetrabutylammonium iodide $(49 \mathrm{mg}, 0.13 \mathrm{mmol})$ was added as a solid, followed by THF (15 mL). 3-buten-1-ol $(0.50 \mathrm{~mL}, 5.8 \mathrm{mmol})$ was added by syringe, and the reaction stirred until all bubbling ceased and the solution turned clear. Bromide VII (315 mg, $0.993 \mathrm{mmol})$ was added by syringe, and the reaction was stirred for $6 \mathrm{~h}$ at room temperature. The reaction mixture was poured into $1 \mathrm{~N} \mathrm{HCl}(40$ $\mathrm{mL})$, and extracted with $\mathrm{Et}_{2} \mathrm{O}(2 \times 50 \mathrm{~mL})$. The combined organic extracts were dried with $\mathrm{MgSO}_{4}$, concentrated in vacuo, and the remaining oil purified by chromatography on silica gel (99:1 hexanes:ether) to give ether 12 (236 mg, $0.765 \mathrm{mmol}, 77 \%$ yield) as a colorless oil.

Compound 12: FTIR (NaCl, thin film): 2949 (s), 2860 (s), 1476 (w), 1255 (m), 1180 (m), 1117 (s), 897 (m), $840(\mathrm{~s}), 777(\mathrm{~m}) \mathrm{cm}^{-1} .{ }^{1} \mathbf{H}$ NMR $\left(400 \mathrm{MHz}, \mathrm{CDCl}_{3}\right)$ : $\square 5.85$ (ddt, $\left.J=17.6,10.4,7.2 \mathrm{~Hz}, 1 \mathrm{H}\right), 5.78$ (s, $1 \mathrm{H}), 5.10(\mathrm{dq}, J=17.2,1.6 \mathrm{~Hz}, 1 \mathrm{H}), 5.04(\mathrm{ddt}, J=10.0,2.4,1.2 \mathrm{~Hz}, 1 \mathrm{H}), 3.96(\mathrm{t}, J=2.0 \mathrm{~Hz}, 2 \mathrm{H}), 3.52(\mathrm{t}, J$ $=7.2 \mathrm{~Hz}, 1 \mathrm{H}), 3.51(\mathrm{t}, J=7.2 \mathrm{~Hz}, 1 \mathrm{H}), 2.82(\mathrm{br} \mathrm{s}, 1 \mathrm{H}), 2.36(\mathrm{qt}, J=6.8,1.4 \mathrm{~Hz}, 2 \mathrm{H}), 1.86(\mathrm{dd}, J=12.0,5.6$ $\mathrm{Hz}, 1 \mathrm{H}), 1.70-1.67$ (m, 1h), 1.48-1.28 (m, 4H), 0.87 (s, 9H), 0.09 (s, 3H), 0.08 (s, 3H). ${ }^{13} \mathbf{C}$ NMR (100 $\left.\mathrm{MHz}, \mathrm{CDCl}_{3}\right)$ : $\square$ 147.9, 135.2, 128.6, 116.4, 87.8, 70.3, 65.6, 52.7, 34.5, 34.1, 26.0, 25.9, 23.8, 18.3, -2.7, -2.8. Anal. Calcd. for $\mathrm{C}_{18} \mathrm{H}_{32} \mathrm{O}_{2} \mathrm{Si}$ C, 70.07; H, 10.45. Found: C, 70.01; H, 10.36. 


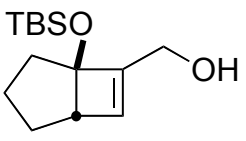

II

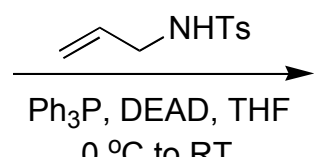

$0{ }^{\circ} \mathrm{C}$ to RT

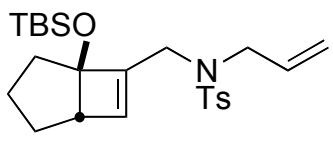

14

A flask was charged with $\mathrm{Ph}_{3} \mathrm{P}(620 \mathrm{mg}, 2.36 \mathrm{mmol})$ and allyl tosylamide $(500 \mathrm{mg}, 2.36 \mathrm{mmol})$. Both were dissolved in THF $(20 \mathrm{~mL})$, and alcohol II $(605 \mathrm{mg}, 2.38 \mathrm{mmol})$ was added by syringe. The reaction was cooled to $0{ }^{\circ} \mathrm{C}$, and DEAD $(0.37 \mathrm{~mL}, 2.35 \mathrm{mmol})$ was added dropwise. After 30 minutes, the reaction was warmed to room temperature and allowed to stir for $24 \mathrm{~h}$. The reaction was diluted with $\mathrm{Et}_{2} \mathrm{O}(100 \mathrm{~mL})$ and washed with water $(75 \mathrm{~mL})$ and brine $(75 \mathrm{~mL})$. The organic layer was dried with $\mathrm{MgSO}_{4}$ and concentrated in vacuo, and purification of the residue by chromatography on silica gel (85:15 hexanes:ether) gave tosyl amide 14 (587 mg, $1.31 \mathrm{mmol}, 55 \%$ yield) as a white solid.

Compound 14: FTIR (NaCl, thin film): 3043 (w), 2955 (s), 2854 (s), 1463 (w), 1350 (m), 1255 (m), 1161 (s), $1111(\mathrm{~m}), 916$ (m), 897 (m), 840 (m), 780 (m), 664 (m) cm ${ }^{-1} .{ }^{1} \mathbf{H}$ NMR (400 MHz, CDCl $): \square 7.70$ (d, J $=8.4 \mathrm{~Hz}, 2 \mathrm{H}), 7.26(\mathrm{~d}, J=8 \mathrm{~Hz}, 2 \mathrm{H}), 5.62(\mathrm{ddt}, J=16.0,10.0,6.8 \mathrm{~Hz}, 1 \mathrm{H}), 5.42(\mathrm{~s}, 1 \mathrm{H}), 5.13(\mathrm{dd}, J=$ 16.4, $1.0 \mathrm{~Hz}, 1 \mathrm{H}), 5.12$ (dd, $J=10.8,1 \mathrm{~Hz}, 1 \mathrm{H}), 3.87$ (d, $J=6.8 \mathrm{~Hz}, 2 \mathrm{H}), 3.75$ (s, 2H), 2.73 (br s, $1 \mathrm{H}), 2.41$ (s, 3H), 1.71-1.63 (m, 2 H), 1.38-1.22 (m, 4H), 0.84 (s, 9H), 0.05 (s, 3H), 0.04 (s, 3H). ${ }^{13}$ C NMR (100 $\mathrm{MHz}_{\mathrm{CDCl}}$ ): $\mathrm{C} 145.5,143.1,137.6,132.8,129.6,129.5,127.3,119.1,87.7,52.3,50.2,42.5,33.7,26.0$, 25.8, 23.7, 21.8, 18.2, -2.6, -2.9. Anal. Calcd. for $\mathrm{C}_{24} \mathrm{H}_{37} \mathrm{NO}_{3} \mathrm{SSi}$ : C, 64.39; H, 8.33; N, 3.13. Found: C, $64.55 ; \mathrm{H}, 8.56 ; \mathrm{N}, 3.00$.

Representative procedure for ring-rearrangement metathesis: A flask was charged with Grubbs' catalyst (4) (10.0 mg, $0.0118 \mathrm{mmol})$, fitted with a condenser, and put under a nitrogen atmosphere. Benzene $(10 \mathrm{~mL})$ was added to dissolve the catalyst, and the cyclobutene $(0.60 \mathrm{mmol})$ was added by syringe, either neat or as a solution in benzene. The reaction was heated to $60{ }^{\circ} \mathrm{C}$ and allowed to stir for $1 \mathrm{~h}$. The reaction was then cooled to room temperature; ethyl vinyl ether $(1 \mathrm{~mL})$ was added to the reaction, and the solution was concentrated in vacuo. The remaining residue was chromatographed on silica gel.

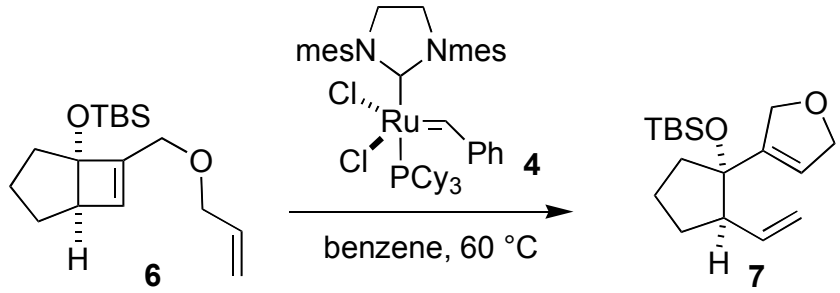

Compound $7^{8}$ : After chromatography (95:5 hexanes:ether), isolated as a colorless oil in $89 \%$ yield. FTIR (NaCl, thin film): 2955 (s), 2856 (s), 1471 (m), 1255 (m), 1105 (m), 1078 (m), 1024 (m), 830 (s), 773 (m) $\mathrm{cm}^{-1} .{ }^{1} \mathbf{H}$ NMR (400 MHz, $\left.\mathrm{CDCl}_{3}\right): \square 5.64-5.55(\mathrm{~m}, 2 \mathrm{H}), 5.00(\mathrm{dt}, J=17.2,1.2 \mathrm{~Hz}, 1 \mathrm{H}), 4.94(\mathrm{br} \mathrm{d}, J=$

\footnotetext{
${ }^{8}$ Compounds 7, 9, and $\mathbf{1 1}$ decomposed within days when stored neat; elemental analysis could not be performed. Attempts at HRMS were unsuccessful.
} 
$10.4 \mathrm{~Hz}, 1 \mathrm{H}), 4.70-4.59$ (m, 4H), 2.55 (br q, $J=6.8 \mathrm{~Hz}, 1 \mathrm{H}), 2.10-1.90$ (m, 3H), 1.83-1.67 (m, $2 \mathrm{H}), 1.55$ (ddt, $J=13.0,9.0,4.8 \mathrm{~Hz}, 1 \mathrm{H}), 0.87(\mathrm{~s}, 9 \mathrm{H}), 0.10$ (s, 3H), $0.06(\mathrm{~s}, 3 \mathrm{H}) .{ }^{13} \mathbf{C ~ N M R}\left(100 \mathrm{MHz}, \mathrm{CDCl}_{3}\right):[$ $144.2,138.9,120.8,114.8,84.0,75.8,75.7,55.8,36.7,29.0,26.2,21.0,18.7,-2.1,-2.9$.

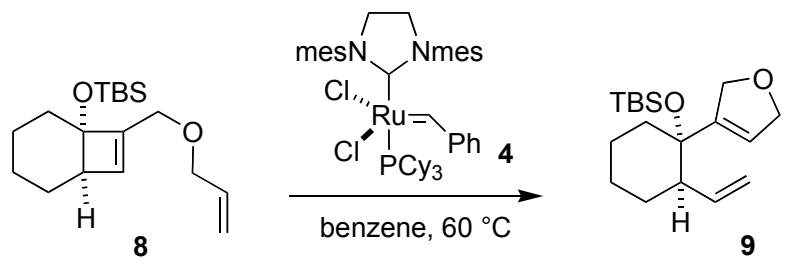

Compound $9^{8}$ : After chromatography (95:5 hexanes:ether), isolated as a colorless oil in $82 \%$ yield. FTIR (NaCl, thin film): 2936 (s), 2860 (s), 1470 (m), 1362 (w), 1255 (m), 1079 (s), 1029 (s), 909 (m), 840 (s), $777(\mathrm{~s}), 676(\mathrm{~m}) \mathrm{cm}^{-1} .{ }^{1} \mathbf{H}$ NMR $\left(400 \mathrm{MHz} \mathrm{CDCl}_{3}\right)$ : $\square 5.90(\mathrm{ddd}, J=17.6,10.4,7.6 \mathrm{~Hz}, 1 \mathrm{H}), 9.65$ (s, $\left.1 \mathrm{H}\right)$, $5.04(\mathrm{dt}, J=17.2,1.0 \mathrm{~Hz}, 1 \mathrm{H}), 5.01$ (dt, $J=10.4,1.0 \mathrm{~Hz}, 1 \mathrm{H}), 4.62$ (br s, 4H), 2.29 (br m, 1H), 2.04-1.97 (m, 1H), 1.89 (ddd, $J=14.0,10.0,4.0 \mathrm{~Hz}, 1 \mathrm{H}), 1.80-1.66(\mathrm{~m}, 2 \mathrm{H}), 1.54-1.39$ (m, 4H), 0.90 (s, $9 \mathrm{H}), 0.09$ (s, 3H), 0.03 (s, 3H). ${ }^{13} \mathrm{C}$ NMR (100 MHz, $\left.\mathrm{CDCl}_{3}\right)$ : $\square$ 145.2, 138.9, 121.4, 115.3, 75.8, 74.8, 74.5, 49.7, $34.4,27.9,26.3,22.4,22.1,18.9,-1.7,-2.8$.
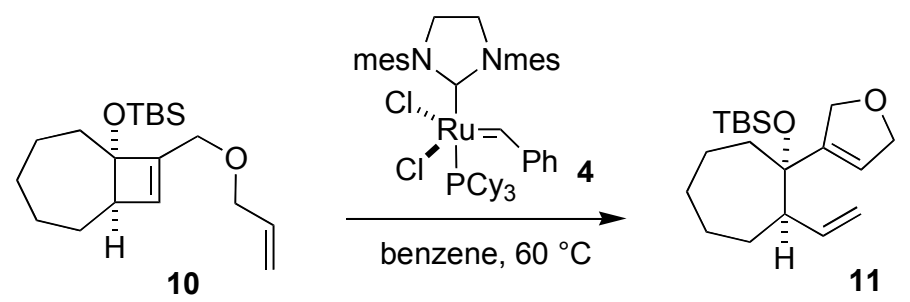

Compound 11 ${ }^{8}$ : After chromatography (95:5 hexanes:ether), isolated as a colorless oil in 82\% yield. FTIR ( NaCl, thin film): 2930 (s), 2854 (s), 1469 (w), 1255 (m), 1086 (m), 1054 (m), 997 (w), 909 (w), 840 (m), $780(\mathrm{~m}) \mathrm{cm}^{-1} .{ }^{1} \mathbf{H}$ NMR $\left(400 \mathrm{MHz}, \mathrm{CDCl}_{3}\right)$ : $\square 5.74(\mathrm{dddd}, J=17.2,10.4,8.4,2.0 \mathrm{~Hz}, 1 \mathrm{H}), 5.63(\mathrm{~d}, J=1.0$ $\mathrm{Hz}, 1 \mathrm{H}), 5.02(\mathrm{dq}, J=18.0,1.0 \mathrm{~Hz}, 1 \mathrm{H}), 5.01$ (dq, $J=10.0,1.0 \mathrm{~Hz}, 1 \mathrm{H}), 4.63$ (s, 4H), 2.44 (br t, $J=8.0$ $\mathrm{Hz}, 1 \mathrm{H}), 2.07(\mathrm{dd}, J=14.8,8.0 \mathrm{~Hz}, 1 \mathrm{H}), 1.98(\mathrm{ddd}, J=14.0,10.0,4.0 \mathrm{~Hz}, 1 \mathrm{H}), 1.87-1.80$ (m, 2H), 1.76-1.64 (m, 2H), 1.57-1.30 (m, 4H), $0.90(\mathrm{~s}, 9 \mathrm{H}), 0.11(\mathrm{~s}, 3 \mathrm{H}), 0.06(\mathrm{~s}, 3 \mathrm{H}) .{ }^{13} \mathrm{C}$ NMR $(100 \mathrm{MHz}$, $\left.\mathrm{CDCl}_{3}\right)$ : $\square 146.4,139.3,121.1,115.2,77.5,75.9,75.6,53.7,40.3,28.8,26.9,26.4,26.1,25.4,21.8,-1.7$, -2.6 .

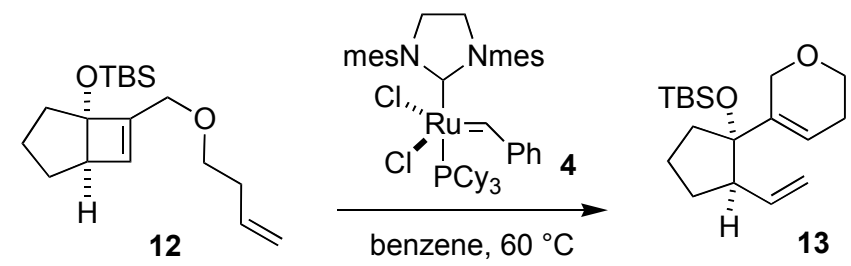

Compound 13: After chromatography (95:5 hexanes:ether), isolated as a colorless oil in $83 \%$ yield. FTIR (NaCl, thin film): 2961 (s), 2936 (s), 2854 (s), 1476 (w), 1262 (m), 1111 (s), 1027 (s), 845 (s), 777 (m), 670 
(w) $\mathrm{cm}^{-1} .{ }^{1} \mathbf{H}$ NMR (400 MHz, CDCl $)$ : $\square 5.74$ (br s, 1H), 5.56 (ddd, $J=17.2,10.4,8.8 \mathrm{~Hz}, 1 \mathrm{H}$ ), 5.02 (br d, $J=16.8 \mathrm{~Hz}, 1 \mathrm{H}), 4.96(\mathrm{dd}, J=10.0,1.0 \mathrm{~Hz}, 1 \mathrm{H}), 4.32(\mathrm{dd}, J=15.6,1.0 \mathrm{~Hz}, 1 \mathrm{H}), 4.16(\mathrm{dq}, J=15.6,1.0$ $\mathrm{Hz}, 1 \mathrm{H}), 3.84(\mathrm{dt}, J=10.4,5.2 \mathrm{~Hz}, 1 \mathrm{H}), 3.65(\mathrm{ddd}, J=12.4,7.6,4.8 \mathrm{~Hz}, 1 \mathrm{H}), 2.55$ (br t, $J=7.6 \mathrm{~Hz}, 1 \mathrm{H})$, 2.31-2.10 (m, 3H), 2.04-1.95 (m, 1H), 1.86-1.71 (m, 3H), 1.62-1.56 (m, 1H), $0.91(\mathrm{~s}, 9 \mathrm{H}), 0.12(\mathrm{~s}, 3 \mathrm{H})$, 0.11 (s, 3H). ${ }^{13} \mathbf{C}$ NMR (125 MHz, $\left.\mathrm{CDCl}_{3}\right)$ : $\square$ 140.0, 139.9, 119.8, 114.5, 87.2, 65.9, 64.3, 54.5, 33.8, 29.4, 26.1, 25.4, 20.5, 18.6, -2.4, -3.1. Anal. Calcd. for $\mathrm{C}_{18} \mathrm{H}_{32} \mathrm{O}_{2} \mathrm{Si}$ : C, 70.07; H, 10.45. Found: C, 69.88; $\mathrm{H}$, 10.49
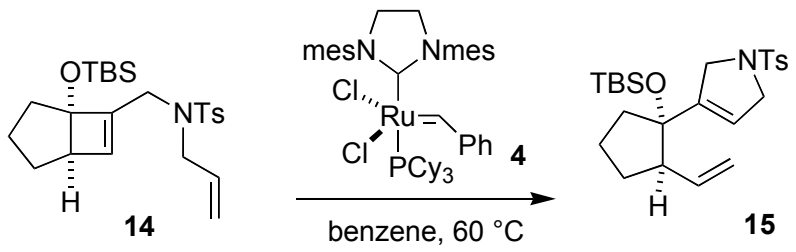

Compound 15: After chromatography (85:15 hexanes:ether), isolated as a pale yellow powder in 95\% yield. FTIR (NaCl, thin film): 2955 (s), 2930 (s), 2860 (s), 1602 (w), 1476 (m), 1350 (s), 1255 (m), 1170 (s), 1111 (m), 1023 (m), 840 (m), 780 (m), 670 (s) cm ${ }^{-1} .{ }^{1} \mathbf{H}$ NMR (400 MHz, CDCl $)$ : 77.71 (d, $J=8 \mathrm{~Hz}$, 2H), 7.31 (d, $J=8 \mathrm{~Hz}, 2 \mathrm{H}), 5.38$ (s, 1H), 5.36 (ddd, $J=17.2,10.4,8.8 \mathrm{~Hz}, 1 \mathrm{H}), 4.89$ (br d, $J=17.2 \mathrm{~Hz}$, 1H), 4.77 (br d, $J=11.0 \mathrm{~Hz}, 1 \mathrm{H}$ ), 4.11 (s, 4H), 2.48 (br q, $J=3.6 \mathrm{~Hz}, 1 \mathrm{H}), 2.43$ (s, 3H), 2.06-2.00 (m, 1H), 1.92-1.64 (m, 4H), 1.48 (tt, $J=9.2,5.6 \mathrm{~Hz}, 1 \mathrm{H}), 0.083$ (s, 9H), 0.01 (s, 3H), -0.21 (s, 3H). ${ }^{13} \mathbf{C}$ NMR (100 $\left.\mathrm{MHz}, \mathrm{CDCl}_{3}\right): \square 143.4,143.2,138.4,134.1,129.8,127.7,119.3,115.0,84.2,55.7,55.2,55.0,35.8,29.0$, 26.1, 21.8, 21.0, 18.6, -2.2, -3.2. Anal. Calcd. for $\mathrm{C}_{24} \mathrm{H}_{37} \mathrm{NO}_{3} \mathrm{SSi}$ : C, 64.39; H, 8.33; N, 3.13. Found: C, $64.18 ; \mathrm{H}, 8.31 ; \mathrm{N}, 2.90$.

General procedure for TBS deprotections: A flask was charged with the silyl ether $(0.50$ mmol), and dissolved in THF (4 mL). TBAF (392 mg, $1.50 \mathrm{mmol}$ ) was added as a solid, and the reaction was stirred until no more starting material remained by TLC (generally 18-24 h). The reaction was poured into $1 \mathrm{~N} \mathrm{HCl}(30 \mathrm{~mL})$, and extracted with ethyl acetate $(2 \times 30 \mathrm{~mL})$. The combined organic extracts were dried with $\mathrm{MgSO}_{4}$ and concentrated in vacuo. The resulting residue was purified by chromatography on silica gel.

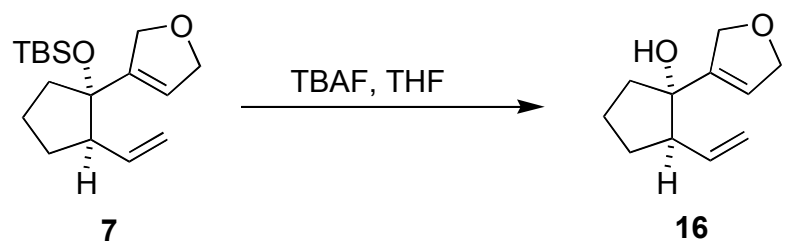

Compound 16: After chromatography (2:1 hexanes:ether), isolated as a colorless oil in $89 \%$ yield. FTIR (NaCl, thin film): 3408 (b), 2955 (s), 2854 (s), 1193 (w), 1079 (s), 1016 (m), 966 (w), 922 (s), 827 (w), 802 (w), $760(\mathrm{w}) \mathrm{cm}^{-1} .{ }^{1} \mathbf{H}$ NMR (400 MHz, $\left.\mathrm{CDCl}_{3}\right)$ : $\square 5.68$ (d, $\left.J=1.6 \mathrm{~Hz}, 1 \mathrm{H}\right), 5.60$ (ddd, $J=17.6,10.4,8.8$ $\mathrm{Hz}, 1 \mathrm{H}), 5.03$ (dt, $J=17.0,1.6 \mathrm{~Hz}, 1 \mathrm{H}), 4.97$ (dd, $J=10.0,1.6 \mathrm{~Hz}, 1 \mathrm{H}), 4.73-4.61$ (m, 4H), 2.58 (br q, $J=$ 
$7.2 \mathrm{~Hz}, 1 \mathrm{H}), 2.15-2.02(\mathrm{~m}, 2 \mathrm{H}), 1.91-1.71(\mathrm{~m}, 3 \mathrm{H}), 1.63-1.55(\mathrm{~m}, 1 \mathrm{H}) .{ }^{13} \mathbf{C} \mathbf{N M R}\left(100 \mathrm{MHz}, \mathrm{CDCl}_{3}\right)$ : 144.0, 138.8, 120.5, 115.1, 82.1, 75.9, 75.3, 53.9, 38.2, 30.1, 21.4. Anal. Calcd. for $\mathrm{C}_{11} \mathrm{H}_{16} \mathrm{O}_{2}$ : C, 73.30; $\mathrm{H}$, 8.95. Found: C, $73.17 ; \mathrm{H}, 9.23$.

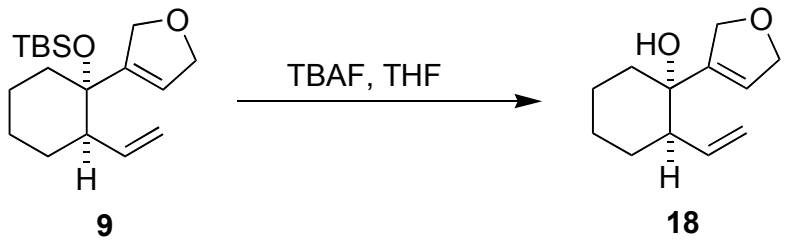

Compound 18: After chromatography (2:1 hexanes:ether), isolated as a colorless oil in $91 \%$ yield. FTIR ( NaCl, thin film): 3414 (b), 2946 (s), 2860 (s), 1457 (w), 1218 (w), 1086 (m), 1016 (m), 909 (m), 809 (w), $758(\mathrm{w}) \mathrm{cm}^{-1} .{ }^{1} \mathbf{H}$ NMR $\left(500 \mathrm{MHz}, \mathrm{CDCl}_{3}\right)$ : $\square 5.87$ (br dt, $\left.J=18.0,9.0 \mathrm{~Hz}, 1 \mathrm{H}\right), 5.70(\mathrm{~s}, 1 \mathrm{H}), 5.10$ (dt, $J=$ $19.0,1.0 \mathrm{~Hz}, 1 \mathrm{H}), 5.07$ (dt, $J=10.0,1.0 \mathrm{~Hz}, 1 \mathrm{H}), 4.72-4.63(\mathrm{~m}, 4 \mathrm{H}), 2.30$ (br q, $J=8.0 \mathrm{~Hz}, 1 \mathrm{H}), 2.00$ (br s, $1 \mathrm{H}), 1.94-1.87(\mathrm{~m}, 2 \mathrm{H}), 1.74-1.68(\mathrm{~m}, 1 \mathrm{H}), 1.61-1.51(\mathrm{~m}, 3 \mathrm{H}), 1.44-1.38(\mathrm{~m}, 1 \mathrm{H}) .{ }^{13} \mathbf{C}$ NMR $(125 \mathrm{MHz}$, $\left.\mathrm{CDCl}_{3}\right): \square$ 145.0, 138.5, 121.3, 116.7, 76.0, 75.1, 72.5, 50.3, 35.5, 28.2, 22.8, 22.3. Anal. Calcd. for $\mathrm{C}_{12} \mathrm{H}_{18} \mathrm{O}_{2}$ : C, 74.19; H, 9.34. Found: C, 73.86; H, 9.38.

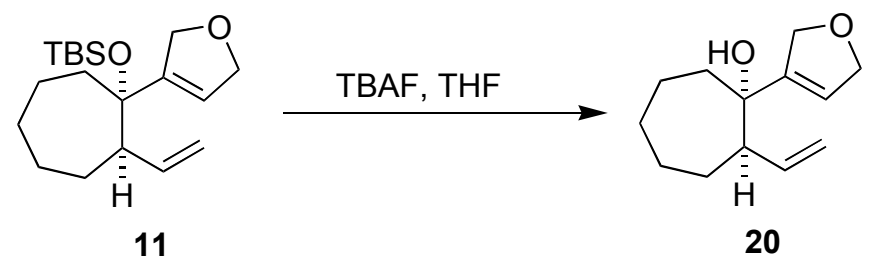

Compound 20: After chromatography (2:1 hexanes:ether), isolated as a colorless oil in $81 \%$ yield. FTIR ( $\mathrm{NaCl}$, thin film): 3433 (b), 2923 (s), 2854 (s), 1457 (m), 1070 (m), 902 (m) cm ${ }^{-1} .{ }^{1} \mathbf{H}$ NMR (400 MHz, $\mathrm{CDCl}_{3}$ ): $\mathrm{\square} 5.75-5.66(\mathrm{~m}, 2 \mathrm{H}), 5.07(\mathrm{br} \mathrm{d}, J=16.8 \mathrm{~Hz}, 1 \mathrm{H}), 5.04(\mathrm{dd}, J=10.0,1.0 \mathrm{~Hz}, 1 \mathrm{H}), 4.67-4.62(\mathrm{~m}$, $4 \mathrm{H}), 2.33$ (br t, $J=9.0 \mathrm{~Hz}, 1 \mathrm{H}), 1.93-1.82(\mathrm{~m}, 3 \mathrm{H}), 1.80-1.68(\mathrm{~m}, 2 \mathrm{H}), 1.65-1.59$ (m, 2H), 1.47-1.40 (m, 2H), 1.34-1.23 (m, 1H). ${ }^{13} \mathbf{C}$ NMR (100 MHz, $\left.\mathrm{CDCl}_{3}\right)$ : $\square$ 146.1, 139.7, 120.7, 115.8, 76.8, 75.7, 75.4, 55.3, 40.8, 30.3, 29.4, 21.9. Anal. Calcd. for $\mathrm{C}_{13} \mathrm{H}_{20} \mathrm{O}_{2}: \mathrm{C}, 74.96 ; \mathrm{H}, 9.68$. Found: C, 75.12; H, 9.90.

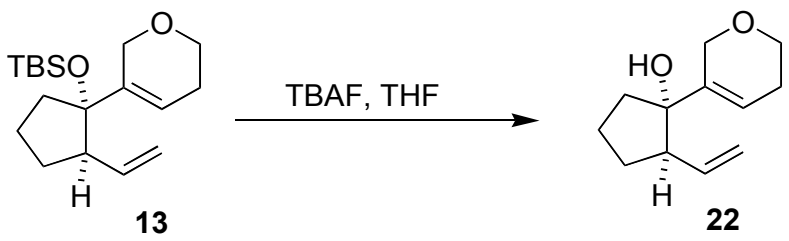

Compound 22: No aqueous workup was performed; after the reaction was complete, the resulting reaction mixture was concentrated in vacuo. The remaining thick, yellow oil was dissolved in a minimal amount of $\mathrm{CH}_{2} \mathrm{Cl}_{2}$ and loaded onto a silica gel column, eluting with 3:1 hexanes:ether. Alcohol 22 was isolated as a colorless oil in 97\% yield. FTIR ( $\mathrm{NaCl}$, thin film): 3328 (s), 2965 (s), 2858 (s), 1634 (m), 1390 (m), 1250 (m), 1099 (s), 1051 (m), 915 (m), 873 (m) cm ${ }^{-1}$. ${ }^{1} \mathbf{H}$ NMR (400 MHz, CDCl $)$ : $\square 5.81$ (dt, $J=3.0,1.6 \mathrm{~Hz}$, 
1H), 5.56 (ddd, $J=16.8,10.4,8.8 \mathrm{~Hz}, 1 \mathrm{H}), 5.01$ (br d, $J=17.2 \mathrm{~Hz}, 1 \mathrm{H}), 4.95$ (dd, $J=10.4,1.6 \mathrm{~Hz}, 1 \mathrm{H}$ ), 4.35 (dq, $J=15.2,2.0 \mathrm{~Hz}, 1 \mathrm{H}), 4.15(\mathrm{dq}, J=15.2,2.0 \mathrm{~Hz}, 1 \mathrm{H}), 3.77$ (dt, $J=10.8,5.6 \mathrm{~Hz}, 1 \mathrm{H}), 3.66$ (ddd, $J$ $=11.2,6.4,5.2 \mathrm{~Hz}, 1 \mathrm{H}), 2.54(\mathrm{td}, J=8.4,3.2 \mathrm{~Hz}, 1 \mathrm{H}), 2.21-2.10(\mathrm{~m}, 3 \mathrm{H}), 2.06(\mathrm{dt}, J=13.2,9.6 \mathrm{~Hz}, 1 \mathrm{H})$, 1.88-1.69 (m, 2H), 1.66-1.53 (m, 2H). ${ }^{13} \mathbf{C}$ NMR (100 MHz, $\left.\mathrm{CDCl}_{3}\right)$ : $\square$ 139.9, 139.7, 119.5, 114.5, 85.0, 65.8, 64.3, 53.0, 35.9, 30.1, 25.4, 20.9. Anal. Calcd. for $\mathrm{C}_{12} \mathrm{H}_{18} \mathrm{O}_{2}$ : C, 74.19; H, 9.34. Found: C, 73.91; H, 9.05 .

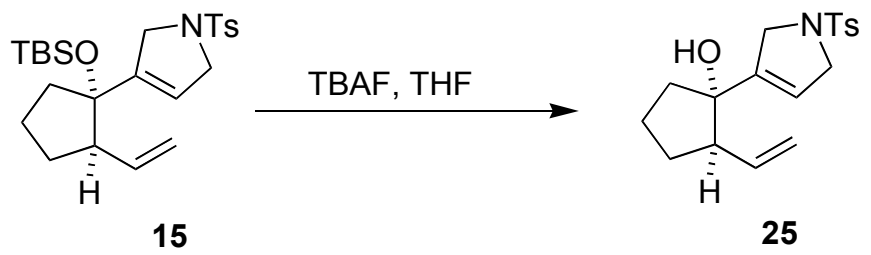

Compound 25: After chromatography (3:2 hexanes:ether), isolated as a thick, yellow oil in $84 \%$ yield. FTIR (NaCl, thin film): 3501 (b), 2957 (s), 2869 (s), 1597 (m), 1471 (m), 1337 (s), 1160 (s), 1102 (m), 912 (m), 814 (m), 667 (s), 601 (s) cm ${ }^{-1} .{ }^{1} \mathbf{H}$ NMR (400 MHz, $\left.\mathrm{CDCl}_{3}\right)$ : 7.71 (d, $\left.J=8 \mathrm{~Hz}, 2 \mathrm{H}\right), 7.32$ (d, $J=8$ Hz, 2H), 5.45 (br s, 1H), 5.35 (ddd, $J=16.8,10.4,8.8 \mathrm{~Hz}, 1 \mathrm{H}), 4.91$ (br d, $J=16.8 \mathrm{~Hz}, 1 \mathrm{H}$ ), 4.78 (dd, $J=$ $10.2,1.6 \mathrm{~Hz}, 1 \mathrm{H}), 4.24-4.19(\mathrm{~m}, 1 \mathrm{H}), 4.14-4.08(\mathrm{~m}, 3 \mathrm{H}), 2.49$ (br q, $J=8.0 \mathrm{~Hz}, 1 \mathrm{H}), 2.43(\mathrm{~s}, 3 \mathrm{H}), 2.10$ (dddd, $J=13.2,10.0,8.0,4.8 \mathrm{~Hz}, 1 \mathrm{H}), 1.95(\mathrm{dt}, J=13.2,9.6 \mathrm{~Hz}, 1 \mathrm{H}), 1.89-1.80(\mathrm{~m}, 1 \mathrm{H}), 1.78-1.67$ (m, $2 \mathrm{H}), 1.53$ (dddd, $J=12.0,8.8,6.6,5.6 \mathrm{~Hz}, 1 \mathrm{H}) .{ }^{13} \mathbf{C}$ NMR $\left(100 \mathrm{MHz}, \mathrm{CDCl}_{3}\right): \square$ 143.5, 142.9, 138.3, 134.1, 129.8, 127.6, 119.5, 115.3, 82.5, 55.2, 54.9, 54.8, 37.7, 30.0, 21.9, 21.4. Anal. Calcd. for $\mathrm{C}_{18} \mathrm{H}_{23} \mathrm{NO}_{3} \mathrm{~S}$ : C, 64.84; H, 6.95; N, 4.20. Found: C, 64.60; H, 6.92; N, 4.03.
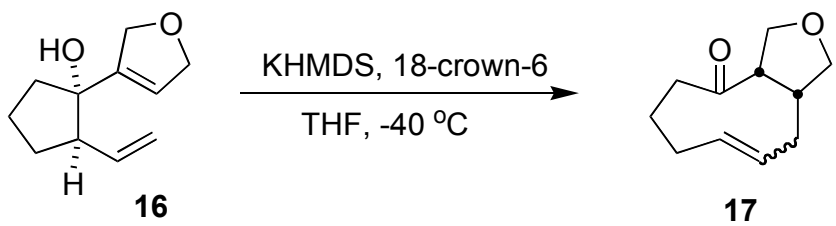

Alcohol 16 (50 mg, $0.277 \mathrm{mmol})$ was added by syringe to a solution of 18 -crown-6 ( $80.0 \mathrm{mg}, 0.30 \mathrm{mmol})$ in THF $(5 \mathrm{~mL})$, and cooled to $-40{ }^{\circ} \mathrm{C}$. KHMDS $(0.60 \mathrm{~mL}, 0.5 \mathrm{M}$ in toluene, $0.30 \mathrm{mmol})$ was added dropwise, and the reaction stirred for $1 \mathrm{~h}$. While still cold, the reaction mixture was poured into $1 \mathrm{~N} \mathrm{HCl}(30$ $\mathrm{mL})$, and extracted with ethyl acetate $(2 \times 30 \mathrm{~mL})$. The combined organic extracts were dried with $\mathrm{MgSO}_{4}$, and concentrated in vacuo. The remaining residue was purified by chromatography (4:1 hexanes:ether) to give cis-17 (27.0 mg, $0.150 \mathrm{mmol}, 54 \%$ yield) as the less polar product, and trans $\mathbf{1 7}$ (13.0 $\mathrm{mg}, 0.072$ $\mathrm{mmol}, 26 \%$ yield) as the more polar product.

Cis-17: FTIR (NaCl, thin film): 3005 (m), 2961 (s), 2873 (s), 1705 (s), 1460 (m), 1369 (m), 1249 (w), 1125 (m), 1098 (m), 1060 (m), 945 (m), 735 (m), 670 (m) cm ${ }^{-1} .{ }^{1} \mathbf{H}$ NMR (500 MHz, $\left.\mathrm{C}_{6} \mathrm{D}_{6}\right)$ : $\square 5.31$ (td, $J=$ 11.0, $6.0 \mathrm{~Hz}, 1 \mathrm{H}), 5.16$ (td, $J=11.0,6.0 \mathrm{~Hz}, 1 \mathrm{H}), 4.14$ (t, $J=8.0 \mathrm{~Hz}, 1 \mathrm{H}), 3.77$ (t, $J=8.0 \mathrm{~Hz}, 1 \mathrm{H}), 3.70$ (t, 
$J=8.0 \mathrm{~Hz}, 1 \mathrm{H}), 3.26(\mathrm{t}, J=8.5 \mathrm{~Hz}, 1 \mathrm{H}), 2.71(\mathrm{dt}, J=10.5,8.0 \mathrm{~Hz}, 1 \mathrm{H}), 2.32-2.19$ (m, 2H), 2.16 (tdd, $J=$ 11.0, 6.0, $2.5 \mathrm{~Hz}, 1 \mathrm{H}), 2.09-1.98(\mathrm{~m}, 2 \mathrm{H}), 1.70-1.62(\mathrm{~m}, 2 \mathrm{H}), 1.44(\mathrm{dd}, J=14.0,6.0 \mathrm{~Hz}, 1 \mathrm{H}), 1.08(\mathrm{~m}$, 1H). ${ }^{13}$ C NMR (100 MHz, $\left.\mathrm{C}_{6} \mathrm{D}_{6}\right)$ : $\square 212.0,130.8,128.6,74.4,70.6,54.2,45.2,41.1,26.7,24.2,22.8$. Anal. Calcd. for $\mathrm{C}_{11} \mathrm{H}_{16} \mathrm{O}_{2}$ : C, 73.30; H, 8.95. Found: C, 73.54; H, 8.97.

Trans-17: FTIR (NaCl, thin film): 2933 (s), 2856 (s), 1696 (s), 1439 (m), 1415 (m), 1365 (w), 1129 (m), 1084 (m), 988 (m), 937 (w) cm ${ }^{-1} .{ }^{1} \mathbf{H}$ NMR (400 MHz, $\mathrm{C}_{6} \mathrm{D}_{6}$ ): 5.00 (dddd, $J=15.2,11.2,4.0,1.6 \mathrm{~Hz}$, 1H), 4.78 (ddd, $J=14.4,10.4,2.8 \mathrm{~Hz}, 1 \mathrm{H}), 4.29$ (t, $J=9.0 \mathrm{~Hz}, 1 \mathrm{H}), 3.81$ (dd, $J=8.8,7.6 \mathrm{~Hz}, 1 \mathrm{H}), 3.74$ (dd, $J=8.4,6.4 \mathrm{~Hz}, 1 \mathrm{H}), 3.36(\mathrm{dd}, J=8.4,5.2 \mathrm{~Hz}, 1 \mathrm{H}), 2.52(\mathrm{td}, J=9.2,8.0 \mathrm{~Hz}, 1 \mathrm{H}), 2.25-2.08(\mathrm{~m}, 3 \mathrm{H})$, $1.92(\mathrm{q}, \mathrm{H}=11.6 \mathrm{~Hz}, 1 \mathrm{H}), 1.81$ (dtd, $J=10.8,2.8,1.6 \mathrm{~Hz}, 1 \mathrm{H}), 1.67-1.64(\mathrm{~m}, 1 \mathrm{H}), 1.40(\mathrm{td}, J=11.6,5.6$ $\mathrm{Hz}, 1 \mathrm{H}), 1.38-1.33$ (m, 2H). ${ }^{13} \mathbf{C}$ NMR (100 MHz, $\left.\mathrm{C}_{6} \mathrm{D}_{6}\right)$ : $\mathrm{N}$ 209.2, 135.8, 131.0, 74.5, 69.8, 55.3, 46.4, 42.8, 34.7, 32.8, 24.8. HRMS Calcd. for $\mathrm{C}_{11} \mathrm{H}_{16} \mathrm{O}_{2}+\mathrm{Na}$ : 203.1048. Found: 203.1049 .

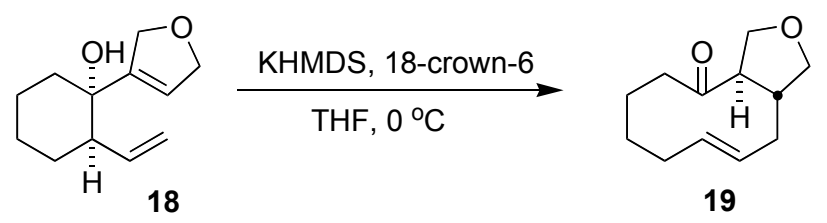

Alcohol $18(54.5 \mathrm{mg}, 0.281 \mathrm{mmol})$ was added to a solution of 18 -crown-6 $(89.0 \mathrm{mg}, 0.336 \mathrm{mmol})$ in THF $(5 \mathrm{~mL})$, and cooled to $0{ }^{\circ} \mathrm{C}$. KHMDS $(0.62 \mathrm{~mL}, 0.5 \mathrm{M}$ in toluene, $0.31 \mathrm{mmol})$ was added dropwise. After 3 $\mathrm{h}$ stirring at $0{ }^{\circ} \mathrm{C}$, methanol $(0.10 \mathrm{~mL})$ was added, and the reaction warmed to room temperature. After an additional $3 \mathrm{~h}$, when reaction mixture converged to one spot by TLC, the reaction was poured into $1 \mathrm{~N} \mathrm{HCl}$ $(20 \mathrm{~mL})$ and extracted with ethyl acetate $(2 \times 40 \mathrm{~mL})$. The combined organic layers were dried with $\mathrm{MgSO}_{4}$ and concentrated in vacuo. The remaining residue was purified by chromatography on silica gel (4:1 hexanes:ether) to give $\mathbf{1 9}$ (44.5 mg, $0.229 \mathrm{mmol}, 82 \%$ yield) as a colorless liquid.

Compound 19: FTIR (NaCl, thin film): 2930 (s), 2854 (s), 1709 (s), 1444 (m), 1381 (m), 1230 (w), 1167

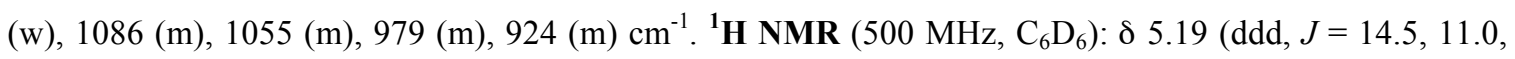
$3.5 \mathrm{~Hz}, 1 \mathrm{H}$ ), 5.09 (dddd, $J=14.0,9.5,4.5,1.0 \mathrm{~Hz}, 1 \mathrm{H}), 3.85$ (t, $J=8.0 \mathrm{~Hz}, 1 \mathrm{H}), 3.84(\mathrm{t}, J=8.0 \mathrm{~Hz}, 1 \mathrm{H})$, $3.69(\mathrm{dd}, J=10.5,8.0 \mathrm{~Hz}, 1 \mathrm{H}), 3.16(\mathrm{dd}, J=10.5,8.0 \mathrm{~Hz}, 1 \mathrm{H}), 2.80$ (qdd, $J=12.0,8.0,3.5 \mathrm{~Hz}, 1 \mathrm{H}), 2.30$ (td, $J=10.5,8.0 \mathrm{~Hz}, 1 \mathrm{H}), 2.05$ (ddd, $J=16.0,9.0,1.5 \mathrm{~Hz}, 1 \mathrm{H}), 2.00-1.92(\mathrm{~m}, 2 \mathrm{H}), 1.87-1.77$ (m, 3H), $1.53(\mathrm{dtt}, J=13.0,6.5,2.0 \mathrm{~Hz}, 1 \mathrm{H}), 1.36(\mathrm{~m}, 1 \mathrm{H}), 1.27(\mathrm{q}, 12.0 \mathrm{~Hz}, 1 \mathrm{H}), 1.08(\mathrm{qt}, J=12.0,2.5 \mathrm{~Hz}, 1 \mathrm{H})$. ${ }^{13}$ C NMR (100 MHz, $\left.\mathrm{C}_{6} \mathrm{D}_{6}\right)$ : $\square$ 209.6, 133.3, 131.2, 72.6, 71.7, 60.0, 51.0, 46.1, 35.2, 33.5, 29.3, 22.9. Anal. Calcd. for $\mathrm{C}_{12} \mathrm{H}_{18} \mathrm{O}_{2}$ : C, 74.19; H, 9.34. Found: C, 73.97; H, 9.48 


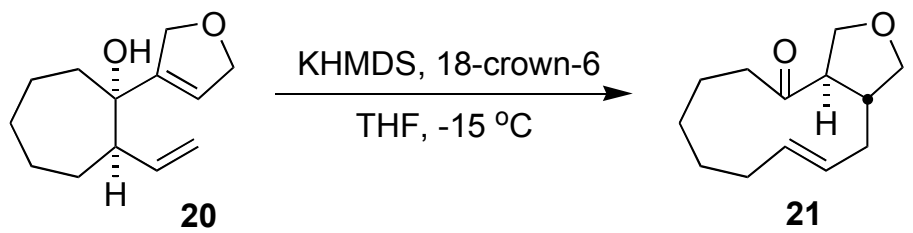

Alcohol 20 (91.5 mg, $0.440 \mathrm{mmol})$ was added to a solution of 18-crown-6 (191 mg, $0.772 \mathrm{mmol})$ in THF $(10 \mathrm{~mL})$ and cooled to $-15{ }^{\circ} \mathrm{C}$. KHMDS $(1.07 \mathrm{~mL}, 0.67 \mathrm{M}$ in toluene, $0.72 \mathrm{mmol})$ was added dropwise, and the reaction stirred for $1 \mathrm{~h}$. Methanol $(1 \mathrm{~mL})$ was added, and the reaction warmed to room temperature. The reaction was stirred for an additional $16 \mathrm{~h}$, until the reaction mixture converged to one spot by TLC. The reaction was poured into $1 \mathrm{~N} \mathrm{HCl}(50 \mathrm{~mL})$, and extracted with ethyl acetate $(50 \mathrm{~mL})$. The organic layer was dried with $\mathrm{MgSO}_{4}$, concentrated in vacuo, and the remaining oil purified by chromatography on silica gel (4:1 hexanes:ether) to give $21(75.0 \mathrm{mg}, 0.360 \mathrm{mmol}, 82 \%)$ as a colorless oil.

Compound 21: FTIR (NaCl, thin film): 2932 (s), 2854 (s), 1705 (s), 1457 (w), 1439 (m), 1377 (m), 1128 (w), $1083(\mathrm{~m}), 1061(\mathrm{~m}), 976(\mathrm{~m}), 926(\mathrm{~m}) \mathrm{cm}^{-1} .{ }^{1} \mathbf{H}$ NMR (500 MHz, $\left.\mathrm{C}_{6} \mathrm{D}_{6}\right)$ : $\square 5.31$ (br ddd, $J=15.0,10.0$, $5.0 \mathrm{~Hz}, 1 \mathrm{H}), 5.14$ (br ddd, $J=15.0,10.0,4.0 \mathrm{~Hz}, 1 \mathrm{H}), 3.97(\mathrm{t}, 8.0 \mathrm{~Hz}, 1 \mathrm{H}), 3.96(\mathrm{t}, J=8.0 \mathrm{~Hz}, 1 \mathrm{H}), 3.44(\mathrm{t}$, $J=8.5 \mathrm{~Hz}, 1 \mathrm{H}), 3.30(\mathrm{t}, J=8.5 \mathrm{~Hz}, 1 \mathrm{H}), 2.91(\mathrm{q}, J=9.0 \mathrm{~Hz}, 1 \mathrm{H}), 2.70(\mathrm{dqd}, J=12.0,9.0,3.0 \mathrm{~Hz}, 1 \mathrm{H})$, $2.56(\mathrm{ddd}, J=15.0,9.5,2.5 \mathrm{~Hz}, 1 \mathrm{H}), 2.24$ (br d, $J=13.0 \mathrm{~Hz}, 1 \mathrm{H}), 2.08(\mathrm{ddd}, J=15.5,8.5,2.5 \mathrm{~Hz}, 1 \mathrm{H})$, $1.76(\mathrm{ddtd}, J=13.0,9.5,6.5,3.0 \mathrm{~Hz}, 1 \mathrm{H}), 1.68$ (q, $J=12.0 \mathrm{~Hz}, 1 \mathrm{H}), 1.64-1.46$ (m, 4H), $1.28-1.21$ (m, 1H), 0.91-0.79 (m, 2H). ${ }^{13} \mathbf{C}$ NMR (100 MHz, $\left.\mathrm{CDCl}_{3}\right)$ : $\square$ 210.7, 131.9, 130.6, 74.2, 72.7, 57.7, 46.1, 43.8, 36.6, 33.9, 25.7, 24.7, 24.1. Anal. Calcd. for $\mathrm{C}_{13} \mathrm{H}_{20} \mathrm{O}_{2}$ : C, 74.96; H, 9.68. Found: C, 74.68; H, 9.43.
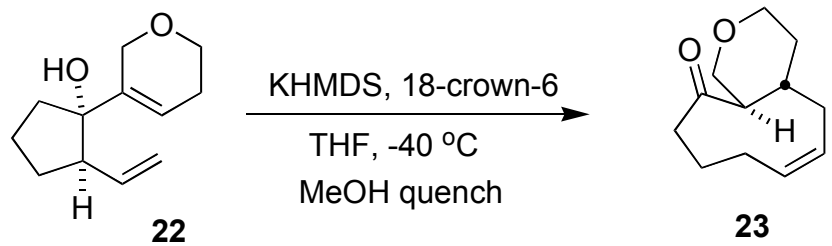

A flask was charged with 18-crown-6 (104 mg, $0.393 \mathrm{mmol})$ and alcohol 22 (47 mg, $0.242 \mathrm{mmol})$. Both were dissolved in THF $(5 \mathrm{~mL})$, and the reaction cooled to $-40{ }^{\circ} \mathrm{C}$. KHMDS $(0.50 \mathrm{~mL}, 0.67 \mathrm{M}$ in toluene, $0.335 \mathrm{mmol}$ ) was added dropwise, and the reaction stirred for $1 \mathrm{~h}$. The reaction was quenched by adding methanol $(0.25 \mathrm{~mL})$, warming to room temperature, and stirring for $16 \mathrm{~h}$. The reaction was poured into $1 \mathrm{~N}$ $\mathrm{HCl}(20 \mathrm{~mL})$ and extracted with ethyl acetate $(2 \times 40 \mathrm{~mL})$. The combined organic extracts were dried with $\mathrm{MgSO}_{4}$, concentrated in vacuo, and the remaining oil purified by chromatography to afford 23 (30.0 mg, $0.154 \mathrm{mmol}, 64 \%$ yield) as a colorless oil.

Compound 23: FTIR (NaCl, thin film): 2928 (s), 2851 (s), 1700 (s), 1457 (w), 1128 (m), 1075 (m), 1001 (m) $\mathrm{cm}^{-1} .{ }^{1} \mathbf{H}$ NMR $\left(500 \mathrm{MHz}, \mathrm{C}_{6} \mathrm{D}_{6}\right)$ : $\square 5.37-5.30(\mathrm{~m}, 2 \mathrm{H}), 3.81(\mathrm{dd}, J=11.0,4.0 \mathrm{~Hz}, 1 \mathrm{H}), 3.77(\mathrm{dd}, J=$ 11.5, 4.0 Hz, 1H), 3.29 (t, $J=11.0 \mathrm{~Hz}, 1 \mathrm{H}), 3.12$ (td, $J=11.5,2.0 \mathrm{~Hz}, 1 \mathrm{H}), 2.47$ (td, $J=10.5,3.5 \mathrm{~Hz}, 1 \mathrm{H})$, $2.02(\mathrm{ddd}, J=13.5,11.0,5.0 \mathrm{~Hz}, 1 \mathrm{H}), 1.92(\mathrm{ddd}, J=13.5,6.5,5.0 \mathrm{~Hz}, 1 \mathrm{H}), 1.83-1.72(\mathrm{~m}, 5 \mathrm{H}), 1.69-1.61$ 
(m, 1H), 1.46-1.39 (m, 1H), $1.21(\mathrm{qd}, J=13.0,5.0 \mathrm{~Hz}, 1 \mathrm{H}), 1.01(\mathrm{br} \mathrm{d}, J=13.0 \mathrm{~Hz}, 1 \mathrm{H}) .{ }^{13} \mathbf{C}$ NMR $(100$ $\left.\mathrm{MHz}, \mathrm{CDCl}_{3}\right)$ : $\square 215.9,130.7,128.9,69.5,66.3,57.6,44.0,40.5,33.4,33.3,25.8,25.3$. Anal. Calcd. for $\mathrm{C}_{12} \mathrm{H}_{18} \mathrm{O}_{2}$ : C, 74.19; H, 9.34. Found: C, 74.36; H, 9.36.

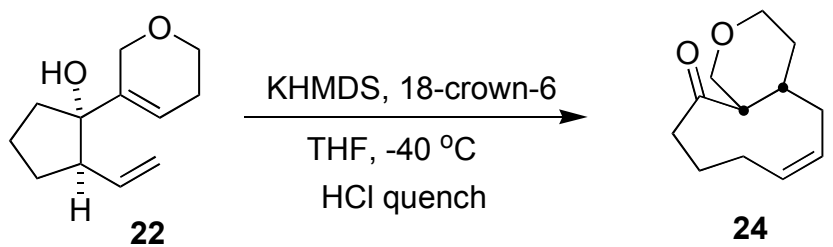

A flask was charged with 18-crown-6 (45.0 mg, $0.170 \mathrm{mmol})$ and alcohol $22(23.7 \mathrm{mg}, 0.122 \mathrm{mmol})$. Both were dissolved in THF $(2 \mathrm{~mL})$, cooled to $-40{ }^{\circ} \mathrm{C}$, and KHMDS $(0.245 \mathrm{~mL}, 0.67 \mathrm{M}$ in toluene, $0.165 \mathrm{mmol})$ was added dropwise. After $1 \mathrm{~h}$, while still cold, the reaction was cannulated into $1 \mathrm{~N} \mathrm{HCl}(20 \mathrm{~mL})$, and extracted with ethyl acetate $(2 \times 40 \mathrm{~mL})$. The organic layer was dried with $\mathrm{MgSO}_{4}$ and concentrated in vacuo. The remaining residue was purified by chromatography on silica gel (4:1 hexanes:ether) to give 24 (14.0 mg, $0.0720 \mathrm{mmol}, 59 \%$ yield) as a colorless oil.

Compound 24: FTIR (NaCl, thin film): 2924 (s), 2851 (s), 1697 (s), 1463 (m), 1436 (w), 1255 (w), 1230 (w), $1144(\mathrm{~m}), 1102(\mathrm{~m}), 726(\mathrm{~m}) \mathrm{cm}^{-1} .{ }^{1} \mathbf{H}$ NMR (500 MHz, $\left.\mathrm{C}_{6} \mathrm{D}_{6}\right): \mathrm{T} .40$ (td, $\left.J=11.0,6.5 \mathrm{~Hz}, 1 \mathrm{H}\right), 5.28$ (td, $J=11.0,6.0 \mathrm{~Hz}, 1 \mathrm{H}), 3.71-3.64$ (m, $2 \mathrm{H}), 3.49$ (ddd, $J=12.0,8.0,3.0 \mathrm{~Hz}, 1 \mathrm{H}), 3.30$ (ddd, $J=11.0,7.0$, $3.5 \mathrm{~Hz}, 1 \mathrm{H}), 2.28-2.15(\mathrm{~m}, 3 \mathrm{H}), 2.08-2.00(\mathrm{~m}, 2 \mathrm{H}), 1.69-1.62(\mathrm{~m}, 4 \mathrm{H}), 1.46$ (ddd, $J=13.0,5.5,2.0 \mathrm{~Hz}$, $1 \mathrm{H}), 1.28(\mathrm{td}, J=7.0,3.5 \mathrm{~Hz}, 1 \mathrm{H}), 1.20$ (tq, $J=13.0,4.0 \mathrm{~Hz}, 1 \mathrm{H}) .{ }^{13} \mathbf{C} \mathbf{N M R}\left(100 \mathrm{MHz}, \mathrm{C}_{6} \mathrm{D}_{6}\right): \square 213.0$, 130.4, 129.3, 66.4, 65.8, 50.7, 38.0, 37.0, 32.3, 29.6, 24.5, 23.9. Anal. Calcd. for $\mathrm{C}_{12} \mathrm{H}_{18} \mathrm{O}_{2}: \mathrm{C}, 74.19 ; \mathrm{H}$, 9.34. Found: C, 74.20; H, 9.30.

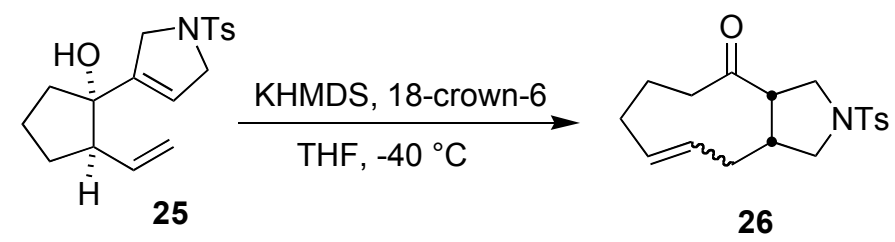

A flask was charged with 18 -crown-6 $(85.0 \mathrm{mg}, 0.322 \mathrm{mmol})$ and alcohol $25(40.0 \mathrm{mg}, 0.120 \mathrm{mmol})$. Both were dissolved in THF $(5 \mathrm{~mL})$, and the reaction was cooled to $-40{ }^{\circ} \mathrm{C}$. KHMDS $(0.48 \mathrm{~mL}, 0.67 \mathrm{M}$ in toluene, $0.32 \mathrm{mmol}$ ) was added dropwise. After stirring for $1 \mathrm{~h}$, the reaction was slowly added to a stirring mixture of THF $(1 \mathrm{~mL})$ and glacial acetic acid $(1 \mathrm{~mL})$ which had been cooled to $-78{ }^{\circ} \mathrm{C}$. The reaction was then poured into $1 \mathrm{~N} \mathrm{HCl}(20 \mathrm{~mL})$ and extracted with ethyl acetate $(40 \mathrm{~mL})$. The organic layer was washed with saturated aqueous $\mathrm{NaHCO}_{3}$, dried with $\mathrm{MgSO}_{4}$, concentrated in vacuo, and the remaining residue chromatographed on silica gel (4:1 hexanes:ether) to give cis-26 (22.0 $\mathrm{mg}, 0.066 \mathrm{mmol}, 55 \%$ yield) as the less polar product, and trans-26 ( $7.0 \mathrm{mg}, 0.021 \mathrm{mmol}, 17 \%$ yield) as the more polar product. 
Cis-26: FTIR (NaCl, thin film): 2999 (m), 2912 (m), 2867 (m), 1710 (s), 1474 (m), 1343 (s), 1126 (s), 1101 (m), 1047 (w), 987 (w), 811 (m), 699 (s) $\mathrm{cm}^{-1} .{ }^{1} \mathbf{H}$ NMR $\left(400 \mathrm{MHz}, \mathrm{C}_{6} \mathrm{D}_{6}\right): \square 7.81(\mathrm{~d}, J=8.0 \mathrm{~Hz}, 2 \mathrm{H}), 6.84(\mathrm{~d}, J=8.0$ $\mathrm{Hz}, 2 \mathrm{H}), 5.16(\mathrm{td}, J=10.8,6.0 \mathrm{~Hz}, 1 \mathrm{H}), 5.05(\mathrm{td}, J=10.8,6.4$ $\mathrm{Hz}, 1 \mathrm{H}), 3.42$ (dt, $J=9.6,7.2 \mathrm{~Hz}, 1 \mathrm{H}), 3.34$ (dd, $J=10.4,7.6$ $\mathrm{Hz}, 1 \mathrm{H}), 2.62(\mathrm{t}, J=9.0 \mathrm{~Hz}, 1 \mathrm{H}), 2.53(\mathrm{q}, J=8.0 \mathrm{~Hz}, 1 \mathrm{H})$, $2.10-1.96(\mathrm{~m}, 3 \mathrm{H}), 1.93-1.85(\mathrm{~m}, 2 \mathrm{H}), 1.86(\mathrm{~s}, 3 \mathrm{H}), 1.71(\mathrm{dt}, J=$ $14.0,10.8 \mathrm{~Hz}, 1 \mathrm{H}), 1.57-1.52(\mathrm{~m}, 1 \mathrm{H}), 1.49(\mathrm{dt}, J=11.6,4.0$ $\mathrm{Hz}, 1 \mathrm{H}), 1.32$ (ddd, $J=14.0,5.6,2.4 \mathrm{~Hz}, 1 \mathrm{H}), 0.98$ (tq, $J=11.6$, $4.0 \mathrm{~Hz}, 1 \mathrm{H}) .{ }^{13} \mathbf{C}$ NMR $\left(100 \mathrm{MHz}, \mathrm{C}_{6} \mathrm{D}_{6}\right): \square 211.5,143.2,134.2$, $129.9,129.7,129.0,128.3,54.8,52.6,50.1,43.2,40.5,27.5$, 24.0, 22.9, 21.4. Anal. Calcd. for $\mathrm{C}_{18} \mathrm{H}_{23} \mathrm{NO}_{3} \mathrm{~S}$ : C, 64.84; $\mathrm{H}$, $6.95 ; \mathrm{N}, 4.20$. Found: C, 64.59; H, 7.23; N, 4.50 .

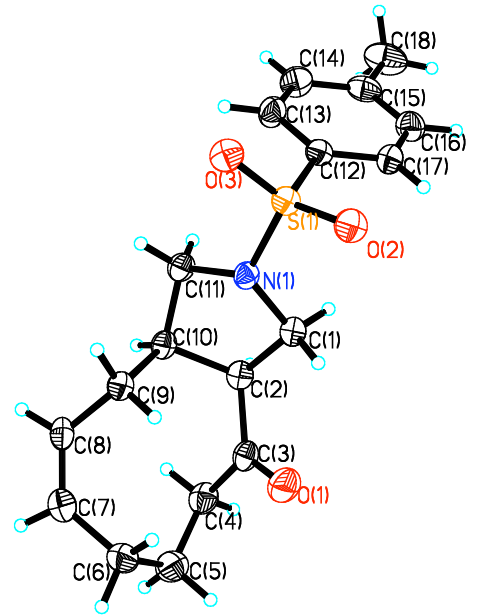

Trans-26: FTIR (NaCl, thin film): 2925 (s), 2862 (s), 1698 (s), 1597 (m), 1454 (m), 1337 (m), 1161 (m), 1037 (m), 814 (s), 737 (m), 665 (m) cm ${ }^{-1} .{ }^{1} \mathbf{H}$ NMR (400 MHz, $\left.\mathrm{C}_{6} \mathrm{D}_{6}\right)$ : $77.78(\mathrm{~d}, J=8 \mathrm{~Hz}, 2 \mathrm{H}), 6.79$ (d, $J=$ $8 \mathrm{~Hz}, 2 \mathrm{H}), 4.71$ (ddd, $J=15.6,11.2,4.0 \mathrm{~Hz}, 1 \mathrm{H}), 4.56(\mathrm{ddd}, J=15.2,12.0,4.4 \mathrm{~Hz}, 1 \mathrm{H}), 3.60$ (t, $J=10.0$ $\mathrm{Hz}, 1 \mathrm{H}), 3.50(\mathrm{dd}, J=10.0,7.6 \mathrm{~Hz}, 1 \mathrm{H}), 3.38(\mathrm{dd}, J=9.6,6.8 \mathrm{~Hz}, 1 \mathrm{H}), 2.81(\mathrm{dd}, J=10.0,4.0 \mathrm{~Hz}, 1 \mathrm{H})$, $2.22(\mathrm{dt}, J=10.0,8.0 \mathrm{~Hz}, 1 \mathrm{H}), 2.12(\mathrm{dt}, J=10.0,4.8 \mathrm{~Hz}, 1 \mathrm{H}), 2.00-1.94(\mathrm{~m}, 1 \mathrm{H}), 1.90-1.80(\mathrm{~m}, 1 \mathrm{H}), 1.86$ (s, 3H), 1.51-1.47 (m, 2H), 1.44-1.34 (m, 2H), 1.33-1.24 (m, 2H). ${ }^{13} \mathbf{C}$ NMR (125 MHz, $\mathrm{CDCl}_{3}$ ): $\mathrm{\square} 210.1$, 143.9, 136.3, 133.2, 130.7, 129.9, 128.0, 54.5, 54.2, 48.5, 44.2, 41.7, 34.9, 32.2, 24.8, 21.8. Anal. Calcd. for $\mathrm{C}_{18} \mathrm{H}_{23} \mathrm{NO}_{3} \mathrm{~S}: \mathrm{C}, 64.84 ; \mathrm{H}, 6.95 ; \mathrm{N}, 4.20$. Found: $\mathrm{C}, 65.03 ; \mathrm{H}, 7.21 ; \mathrm{N}, 4.47$.

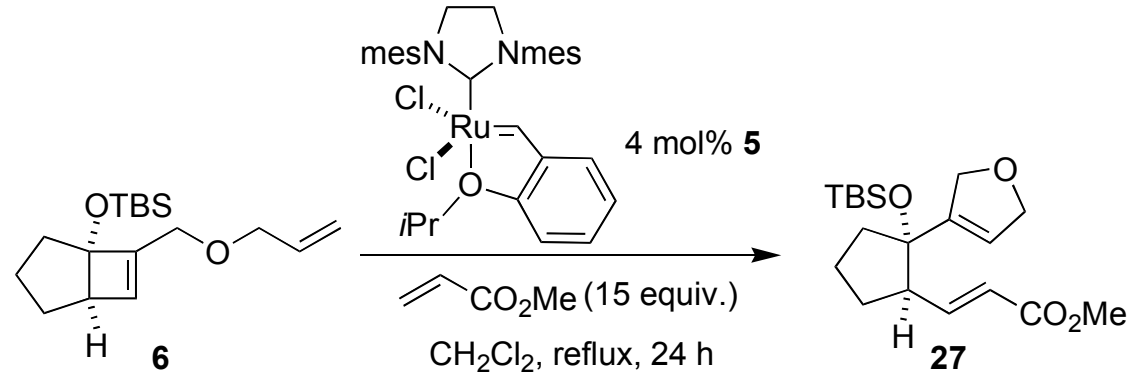

A flask with a reflux condenser was charged with ruthenium catalyst $5(5.0 \mathrm{mg}, 0.0080 \mathrm{mmol})$, which was dissolved in $\mathrm{CH}_{2} \mathrm{Cl}_{2}(5 \mathrm{~mL})$. Cyclobutene $6(56.0 \mathrm{mg}, 0.190 \mathrm{mmol})$ was added by syringe, followed by methyl acrylate $(0.26 \mathrm{~mL}, 2.9 \mathrm{mmol})$. The reaction was refluxed for $24 \mathrm{~h}$; after cooling to room temperature, ethyl vinyl ether $(1 \mathrm{~mL})$ was added, and the reaction concentrated in vacuo. The remaining residue was purified by chromatography on silica gel (3:1 hexanes: $\mathrm{CH}_{2} \mathrm{Cl}_{2}$ to 1:1 hexanes: $\mathrm{CH}_{2} \mathrm{Cl}_{2}$ ) to afford ester 27 (58.0 mg, $0.164 \mathrm{mmol}, 86 \%$ yield) as a colorless oil. 
Compound 27: FTIR (NaCl, thin film): 2955 (s), 2854 (s), 1727 (s), 1658 (w), 1438 (w), 1255 (m), 1104 (w), 1035 (m), 840 (m), 777 (m) cm ${ }^{-1} .{ }^{1} \mathbf{H}$ NMR (400 MHz, $\mathrm{CDCl}_{3}$ ): $\square 6.71$ (dd, $\left.J=15.6,9.0 \mathrm{~Hz}, 1 \mathrm{H}\right), 5.78$ (dd, 15.8, 1.0 Hz, 1H), $5.64(\mathrm{t}, J=1.8 \mathrm{~Hz}, 1 \mathrm{H}), 4.69-4.61(\mathrm{~m}, 3 \mathrm{H}), 4.56-4.51(\mathrm{~m}, 1 \mathrm{H}), 3.71(\mathrm{~s}, 3 \mathrm{H}), 2.70$ (br q, $J=6.0 \mathrm{~Hz}, 1 \mathrm{H}), 2.14-2.05(\mathrm{~m}, 3 \mathrm{H}), 1.89-1.77(\mathrm{~m}, 2 \mathrm{H}), 1.60-1.54(\mathrm{~m}, 1 \mathrm{H}), 0.89(\mathrm{~s}, 9 \mathrm{H}), 0.10(\mathrm{~s}, 3 \mathrm{H})$, 0.05 (s, 3H). ${ }^{13} \mathbf{C}$ NMR (100 MHz, $\left.\mathrm{CDCl}_{3}\right): \square 166.9,148.9,143.2,121.7,120.9,83.9,75.8,75.5,54.6,51.7$, 36.8, 29.0, 26.1, 21.1, 18.7, -2.2, -3.0. Anal. Calcd. for $\mathrm{C}_{19} \mathrm{H}_{32} \mathrm{O}_{4} \mathrm{Si}$ : C, 64.73; H, 9.15. Found: C, 64.86; H, 9.11.

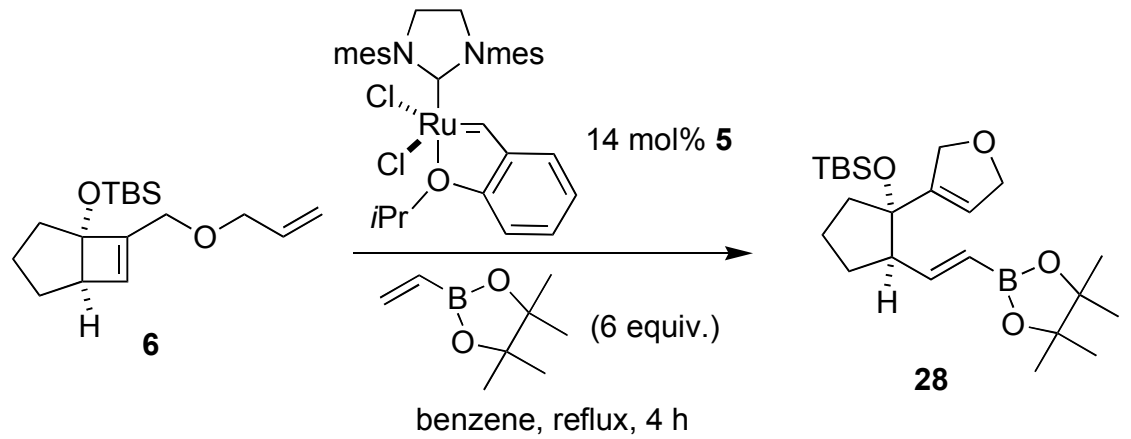

A flask with a reflux condenser was charged with ruthenium catalyst $5(17.0 \mathrm{mg}, 0.0271 \mathrm{mmol})$, which was dissolved in benzene $(10 \mathrm{~mL})$. Cyclobutene $6(65.0 \mathrm{mg}, 0.220 \mathrm{mmol})$ was added, followed by a solution of 4,4,5,5-tetramethyl-2-vinyl-[1,3,2]dioxaborolane $(203 \mathrm{mg}, 1.32 \mathrm{mmol})$ in benzene $(1.8 \mathrm{~mL})$. The reaction was refluxed for $12 \mathrm{~h}$. After cooling to room temperature, ethyl vinyl ether $(1 \mathrm{~mL})$ was added to the reaction, and the reaction mixture concentrated in vacuo. The remaining residue was purified by chromatography on silica gel (95:4:1 hexanes:ether:triethylamine to 90:9:1 hexanes:ether:triethylamine) to afford boronic ester $\mathbf{2 8}$ (72.0 $\mathrm{mg}, 0.171 \mathrm{mmol}, 78 \%$ yield) as a colorless oil.

Compound 28: FTIR (NaCl, thin film): 2955 (s), 2856 (m), 1635 (m), 1476 (m), 1360 (s), 1324 (m), 1145 (m), 1109 (w), 835 (m), 773 (w) cm ${ }^{-1} .{ }^{1} \mathbf{H}$ NMR (400 MHz, $\mathrm{CDCl}_{3}$ ): $\square 6.36$ (dd, $\left.J=18.0,8.8 \mathrm{~Hz}, 1 \mathrm{H}\right), 5.63$ (br s, 1H), 5.42 (d, $J=18.0 \mathrm{~Hz}, 1 \mathrm{H}), 4.73-4.61(\mathrm{~m}, 4 \mathrm{H}), 2.70-2.63$ (m, 1H), 2.16-2.05 (m, 2H), 2.01-1.92 (m, 1H), 1.86-1.74 (m, 2H), 1.65-1.59 (m, 1H), 1.28 (s, 6H), 1.27 (s, 6H), 0.89 (s, 9H), $0.12(\mathrm{~s}, 3 \mathrm{H}), 0.08$ (s, 3H). ${ }^{13}$ C NMR (100 MHz, $\left.\mathrm{CDCl}_{3}\right): \square 153.8,143.7,121.0,84.1,83.2,76.0,75.8,75.6,57.9,36.7,29.1$, 26.2, 25.1, 25.0, 21.2, 18.7, -2.1, -2.9. The carbon $\square$ to the boron could not be seen. HRMS Calcd. for $\mathrm{C}_{23} \mathrm{H}_{41} \mathrm{BO}_{4} \mathrm{Si}+\mathrm{Na}: 443.2765$. Found: 443.2774 .

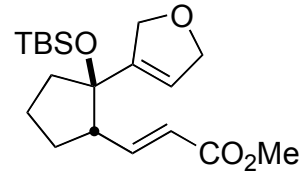

27

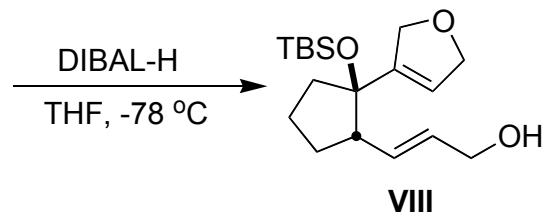

VIII

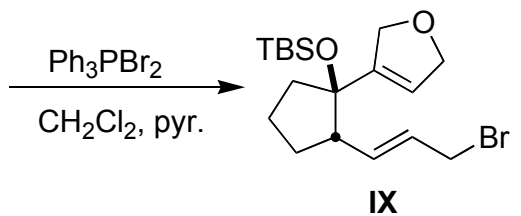

IX 
A solution of ester $27(70.0 \mathrm{mg}, 0.198 \mathrm{mmol})$ in THF (3 mL) was made, and cooled to $-78{ }^{\circ} \mathrm{C}$. DIBAL-H $(0.10 \mathrm{~mL}, 0.59 \mathrm{mmol})$ was added dropwise, and the reaction stirred for $5 \mathrm{~h}$. The reaction, while still cold, was poured into saturated aqueous sodium potassium tartrate $(50 \mathrm{~mL})$, stirred until the solution turned clear, and extracted with ether $(2 \times 75 \mathrm{~mL})$. The combined organic extracts were dried with $\mathrm{MgSO}_{4}$, concentrated in vacuo, and the remaining residue purified by chromatography on silica gel (7:3 ether:hexanes) to give alcohol VIII (53.0 mg, $0.163 \mathrm{mmol}, 82 \%$ yield) as a colorless oil.

Compound VIII: FTIR (NaCl, thin film): 3419 (b), 2953 (s), 2854 (s), 1472 (m), 1252 (m), 1105 (w), 1020 (w), $890(\mathrm{w}), 773(\mathrm{~m}) \mathrm{cm}^{-1}$. ${ }^{1} \mathbf{H}$ NMR (400 MHz, $\left.\mathrm{CDCl}_{3}\right)$ : $\square 5.64$ (dtd, J=15.6, 6.0, $\left.1.0 \mathrm{~Hz}, 1 \mathrm{H}\right), 5.62$ (s, 1H), 5.47 (dd, $J=15.2,8.4 \mathrm{~Hz}, 1 \mathrm{H}), 4.71-4.57(\mathrm{~m}, 4 \mathrm{H}), 4.06$ (d, $J=6.0 \mathrm{~Hz}, 2 \mathrm{H}), 2.59$ (br q, $J=6.8 \mathrm{~Hz}$, 1H), 2.13-2.02 (m, 2H), 1.99-1.92 (m, 1H), 1.89-1.72 (m, 2H), 1.54 (ddt, J=13.6, 9.6, 5.6 Hz, 1H), 0.89 (s, 9H), 0.12 (s, 3H), 0.07 (s, 3H). ${ }^{13} \mathbf{C}$ NMR (100 MHz, $\left.\mathrm{CDCl}_{3}\right)$ : $\square$ 144.2, 132.7, 129.6, 120.8, 84.1, 75.8, 75.7, 63.8, 54.5, 36.8, 29.3, 26.2, 21.1, 18.7, -2.0, -2.9. Anal. Calcd. for $\mathrm{C}_{18} \mathrm{H}_{32} \mathrm{O}_{3} \mathrm{Si}$ : C, 66.62; H, 9.94. Found: C, 66.46; H, 10.01.

A solution of $\mathrm{Ph}_{3} \mathrm{PBr}_{2}(820 \mathrm{mg}, 1.94 \mathrm{mmol})$ in $\mathrm{CH}_{2} \mathrm{Cl}_{2}(20 \mathrm{~mL})$ was cooled to $0{ }^{\circ} \mathrm{C}$. A second solution of alcohol VIII (366 mg, $1.13 \mathrm{mmol})$ and pyridine $(0.18 \mathrm{~mL}, 2.3 \mathrm{mmol})$ in $15 \mathrm{~mL} \mathrm{CH}_{2} \mathrm{Cl}_{2}$ was cannulated into the first solution, and stirred for $2 \mathrm{~h}$ at $0{ }^{\circ} \mathrm{C}$. The solvent was removed in vacuo and the remaining residue purified by column chromatography (95:5 hexanes:ether) to give bromide IX (392 $\mathrm{mg}, 1.01 \mathrm{mmol}, 89 \%$ yield) as a colorless oil.

Compound IX: FTIR (NaCl, thin film): 2955 (s), 2855 (s), 1471 (m), 1359 (w), 1255 (s), 1201 (m), 1107 (m), $964(\mathrm{~m}), 891(\mathrm{M}), 836(\mathrm{~s}), 773(\mathrm{~s}) \mathrm{cm}^{-1} .{ }^{1} \mathbf{H}$ NMR (400 MHz, $\left.\mathrm{CDCl}_{3}\right)$ : $\square 5.71(\mathrm{dt}, J=15.6,8.0 \mathrm{~Hz}$, $1 \mathrm{H}), 5.64(\mathrm{~d}, J=1.6 \mathrm{~Hz}, 1 \mathrm{H}), 5.56(\mathrm{dd}, J=15.2,8.4 \mathrm{~Hz}, 1 \mathrm{H}), 4.72-4.58(\mathrm{~m}, 4 \mathrm{H}), 3.94(\mathrm{~d}, J=7.6 \mathrm{~Hz}, 1 \mathrm{H})$, 2.62 (br q, $J=7.6 \mathrm{~Hz}, 1 \mathrm{H}), 2.14-1.94(\mathrm{~m}, 3 \mathrm{H}), 1.91-1.72(\mathrm{~m}, 2 \mathrm{H}), 1.56(\mathrm{ddt}, J=12.8,9.6,5.6 \mathrm{~Hz}, 1 \mathrm{H})$, 0.90 (s, 9H), 0.13 (s, 3H), 0.09 (s, 3H). $\left.{ }^{13} \mathbf{C ~ N M R ~ ( 1 0 0 ~ M H z , ~} \mathrm{CDCl}_{3}\right): \square$ 143.9, 136.4, 126.7, 121.2, 84.1, 75.8, 75.7, 54.4, 36.9, 33.5, 28.9, 26.2, 21.1, 18.7, -2.0, -2.9. Anal. Calcd. for $\mathrm{C}_{18} \mathrm{H}_{31} \mathrm{BrO}_{2} \mathrm{Si}$ : C, 55.80; H, 8.07. Found: C, 55.60; H, 7.92.

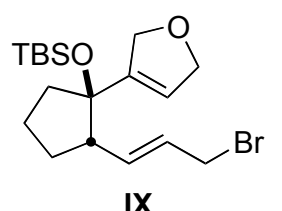

IX

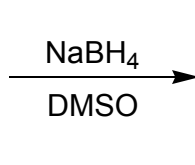

To a suspension of $\mathrm{NaBH}_{4}(42.0 \mathrm{mg}, 1.11 \mathrm{mmol})$ in DMSO $(1.5 \mathrm{~mL})$ was added a solution of bromide $\mathbf{I X}$ $(142 \mathrm{mg}, 0.367 \mathrm{mmol})$ in DMSO $(1.5 \mathrm{~mL})$. After stirring for $1 \mathrm{~h}$, the reaction was poured into $1 \mathrm{~N} \mathrm{HCl}(20$ $\mathrm{mL})$, stirring until all bubbling ceased, and extracted with ether $(40 \mathrm{~mL})$. The organic layer was dried with 
$\mathrm{MgSO}_{4}$, concentrated in vacuo, and the remaining residue purified by chromatography on silica gel to give 29 (89.0 $\mathrm{mg}, 0.208 \mathrm{mmol}, 79 \%)$ as a colorless oil.

Compound 29: FTIR (NaCl, thin film): 2956 (s), 2856 (s), 1472 (m), 1252 (m), 1104 (m), 1079 (m), 964 (w), 835 (s), 773 (s) $\mathrm{cm}^{-1} .{ }^{1} \mathbf{H}$ NMR (400 MHz, $\mathrm{CDCl}_{3}$ ): $\square 5.61$ (s, 1H), 5.44 (dq, $\left.J=15.2,6.4 \mathrm{~Hz}, 1 \mathrm{H}\right)$, $5.22(\mathrm{ddd}, J=15.2,8.4,1.6 \mathrm{~Hz}, 1 \mathrm{H}), 4.72-4.60(\mathrm{~m}, 4 \mathrm{H}), 2.53$ (br q, $J=6.8 \mathrm{~Hz}, 1 \mathrm{H}), 2.10-2.03(\mathrm{~m}, 2 \mathrm{H})$, $1.97-1.93(\mathrm{~m}, 1 \mathrm{H}), 1.87-1.71(\mathrm{~m}, 2 \mathrm{H}), 1.65$ (br d, $J=6.4 \mathrm{~Hz}, 3 \mathrm{H}), 1.52$ (ddt, $J=13.6,9.6,5.6 \mathrm{~Hz}, 1 \mathrm{H})$, 0.90 (s, 9H), 0.12 (s, 3H), 0.08 (s, 3H). ${ }^{13}$ C NMR (100 MHz, CDCl $)$ : $\square$ 144.4, 131.5, 125.4, 120.5, 84.2, 75.8, 75.7, 54.8, 36.7, 29.4, 26.2, 21.0, 18.7, 18.4, -2.1, -2.9. Anal. Calcd. for $\mathrm{C}_{18} \mathrm{H}_{32} \mathrm{O}_{2} \mathrm{Si}: \mathrm{C}, 70.07$; $\mathrm{H}$, 10.45. Found: C, 70.08; H, 10.74 .

To a solution of silyl ether $29(88.0 \mathrm{mg}, 0.285 \mathrm{mmol})$ in THF $(2 \mathrm{~mL})$ was added solid TBAF (260 mg, 1.00 $\mathrm{mmol}$ ). After $16 \mathrm{~h}$, the reaction was concentrated in vacuo, and the remaining residue purified by chromatography on silica gel (7:3 hexanes:ether) to give alcohol $\mathbf{X}(53.0 \mathrm{mg}, 0.273 \mathrm{mmol}, 96 \%$ yield) as a colorless oil.

Compound X: FTIR (NaCl, thin film): 3419 (b), 2958 (s), 2854 (s), 1448 (m), 1377 (w), 1067 (m), 964 (m), 895 (m), 802 (w), 749 (w) cm ${ }^{-1} .{ }^{1} \mathbf{H}$ NMR (400 MHz, CDCl $)$ : $\square 5.70$ (br s, 1H), 5.50 (dq, J = 15.2, 6.4 $\mathrm{Hz}, 1 \mathrm{H}), 5.24$ (ddd, $J=15.6,8.8,1.2 \mathrm{~Hz}, 1 \mathrm{H}), 4.78-4.61$ (m, 4H), 2.55 (br q, $J=7.6 \mathrm{~Hz}, 1 \mathrm{H}$ ), 2.15-2.04 $(\mathrm{m}, 2 \mathrm{H}), 1.93-1.74(\mathrm{~m}, 3 \mathrm{H}), 1.68(\mathrm{dd}, J=8.8,1.2 \mathrm{~Hz}, 3 \mathrm{H}), 1.62-1.55(\mathrm{~m}, 1 \mathrm{H}) .{ }^{13} \mathbf{C}$ NMR $(100 \mathrm{MHz}$, $\left.\mathrm{CDCl}_{3}\right): \square 144.3,131.4,126.1,120.6,82.5,76.0,75.5,54.3,38.4,30.5,21.3,18.3$. Anal. Calcd. for $\mathrm{C}_{12} \mathrm{H}_{18} \mathrm{O}_{2}$ : C, 74.19; H, 9.34. Found: C, 73.95; H, 9.62.

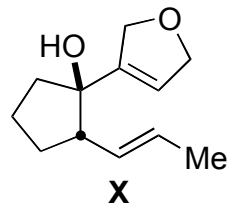

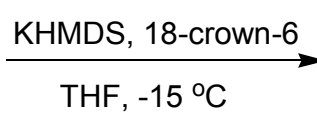

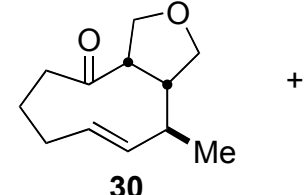

30

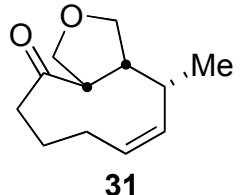

31

To a solution of 18-crown-6 (54.0 mg, $0.204 \mathrm{mmol})$ and alcohol $\mathbf{X}(29.0 \mathrm{mg}, 0.149 \mathrm{mmol})$, cooled to $-15{ }^{\circ} \mathrm{C}$, was added KHMDS $(0.30 \mathrm{~mL}, 0.67 \mathrm{M}$ in toluene, $0.20 \mathrm{mmol})$ dropwise. After stirring for $1 \mathrm{~h}$, the reaction was poured into $1 \mathrm{~N} \mathrm{HCl}(20 \mathrm{~mL})$ and extracted with ethyl acetate $(2 \times 40 \mathrm{~mL})$. The combined organic extracts were dried with $\mathrm{MgSO}_{4}$, concentrated in vacuo, and the remaining residue purified by chromatography on silica gel ( $8: 2$ hexanes:ether) to give $\mathbf{3 0}(13.4 \mathrm{mg}, 0.0690 \mathrm{mmol}, 46 \%$ yield) as the less polar component and $\mathbf{3 1}$ (7.3 $\mathrm{mg}, 0.038 \mathrm{mmol}, 25 \%$ yield) as the more polar component.

Compound 30: FTIR (NaCl, thin film): 2928 (s), 2869 (s), 1697 (s), 1455 (w), 1416 (w), 1363 (m), 1131 (m), 1100 (m), 985 (m), 944 (m), 834 (w) cm ${ }^{-1} .{ }^{1} \mathbf{H}$ NMR (400 MHz, $\mathrm{C}_{6} \mathrm{D}_{6}$ ): $\square 4.98$ (ddd, $J=15.6,11.6,4.0$ Hz, 1H), 4.63 (dd, $J=15.6,10.0 \mathrm{~Hz}, 1 \mathrm{H}), 4.194$ (t, $J=8.8 \mathrm{~Hz}, 1 \mathrm{H}), 3.87$ (t, $J=8.0 \mathrm{~Hz}, 1 \mathrm{H}), 3.44$ (t, $J=8.0$ 
$\mathrm{Hz}, 1 \mathrm{H}), 2.63(\mathrm{td}, J=10.0,8.0 \mathrm{~Hz}, 1 \mathrm{H}), 2.19-2.14(\mathrm{~m}, 1 \mathrm{H}), 2.10-1.94(\mathrm{~m}, 3 \mathrm{H}), 1.69-1.66(\mathrm{~m}, 2 \mathrm{H})$, $1.38-1.29(\mathrm{~m}, 3 \mathrm{H}), 0.69$ (d, $J=6.4 \mathrm{~Hz}, 3 \mathrm{H}) .{ }^{13} \mathbf{C}$ NMR $\left(100 \mathrm{MHz}, \mathrm{C}_{6} \mathrm{D}_{6}\right)$ : $\square 210.1,136.7,133.7,73.3,71.2$, 55.6, 53.8, 43.8, 39.8, 32.7, 25.0, 19.5. Anal. Calcd. for $\mathrm{C}_{12} \mathrm{H}_{18} \mathrm{O}_{2}$ : C, 74.19; H, 9.34. Found: C, 74.14; $\mathrm{H}$, 9.30 .

Compound 31: FTIR (NaCl, thin film): 2956 (s), 2866 (s), 1701 (s), 1457 (m), 1365 (m), 1128 (m), 1079 (m), $934(\mathrm{w}), 760(\mathrm{~m}) \mathrm{cm}^{-1} .{ }^{1} \mathbf{H}$ NMR (400 MHz, $\left.\mathrm{C}_{6} \mathrm{D}_{6}\right)$ : $\square 5.30$ (td, $\left.J=10.0,7.6 \mathrm{~Hz}, 1 \mathrm{H}\right), 5.07$ (t, $J=7.6$ $\mathrm{Hz}, 1 \mathrm{H}), 4.28(\mathrm{t}, J=8.0 \mathrm{~Hz}, 1 \mathrm{H}), 3.74(\mathrm{t}, J=8.8 \mathrm{~Hz}, 1 \mathrm{H}), 3.72(\mathrm{t}, J=8.4 \mathrm{~Hz}, 1 \mathrm{H}), 3.57(\mathrm{t}, J=9.2 \mathrm{~Hz}, 1 \mathrm{H})$, $2.70(\mathrm{ddd}, J=10.4,6.4,3.2 \mathrm{~Hz}, 1 \mathrm{H}), 2.58$ (dt, $J=10.8,6.8 \mathrm{~Hz}, 1 \mathrm{H}), 2.26$ (tdd, $J=10.4,7.2,3.6 \mathrm{~Hz}, 1 \mathrm{H}$ ), 1.89 (br m, $4 \mathrm{H}), 1.73-1.69(\mathrm{~m}, 1 \mathrm{H}), 1.41$ (br s, $1 \mathrm{H}), 0.67$ (d, $J=6.8 \mathrm{~Hz}, 3 \mathrm{H}) .{ }^{13} \mathbf{C}$ NMR $\left(125 \mathrm{MHz}, \mathrm{C}_{6} \mathrm{D}_{6}\right.$, $\left.50{ }^{\circ} \mathrm{C}\right)$ : $\square 209.7,132.2,129.4,69.3,67.6,54.4,49.4,41.6,29.9,27.3,24.1,21.3$. Anal. Calcd. for $\mathrm{C}_{12} \mathrm{H}_{18} \mathrm{O}_{2}$ : C, 74.19; H, 9.34. Found: C, 73.99; H, 9.32.
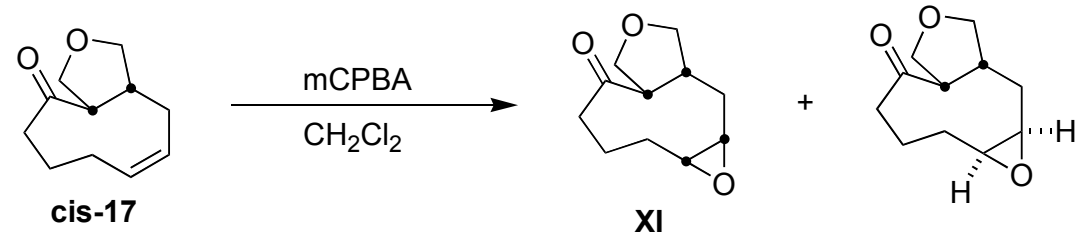

To a solution of cis-17 $(28.0 \mathrm{mg}, 0.155 \mathrm{mmol})$ in $\mathrm{CH}_{2} \mathrm{Cl}_{2}(3 \mathrm{~mL})$ was added mCPBA (110 mg, 0.637 $\mathrm{mmol}$ ) as a solid. The reaction was stirred at room temperature for 16 hours, after which it was diluted with $\mathrm{CHCl}_{3}(20 \mathrm{~mL})$ and washed with saturated aqueous $\mathrm{NaHCO}_{3}(2 \times 20 \mathrm{~mL})$. The organic layer was dried with $\mathrm{MgSO}_{4}$, concentrated in vacuo, and the remaining solid purified by chromatography on silica gel (7:3 hexanes:ether) to give epoxide XI and its minor diastereomer as a 7.5:1 ratio, inseparable by chromatography ( $23.0 \mathrm{mg}, 0.117 \mathrm{mmol}, 75 \%$ overall yield.) The mixture of diastereomers was dissolved in hot ethyl acetate $(0.3 \mathrm{~mL})$, and hot hexanes $(4 \mathrm{~mL})$ was added, and the solution allowed to cool at $-20{ }^{\circ} \mathrm{C}$ over one week. A small amount of the major diastereomer $(5.0 \mathrm{mg}, 0.026 \mathrm{mmol})$ crystallized out of the solution as white needles. X-ray quality crystals were grown from this crop by recrystallization from ether/hexanes.

Compound XI: FTIR (NaCl, thin film): 2924 (s), 2854 (s), 1704 (s), 1453 (m), 1372 (w), 1095 (m), 942 (m), 773 (m), 672 (w) $\mathrm{cm}^{-1} .{ }^{1} \mathbf{H}$ NMR $\left(500 \mathrm{MHz}, \mathrm{C}_{6} \mathrm{D}_{6}\right): \square 4.03(\mathrm{t}, J=8.0 \mathrm{~Hz}, 1 \mathrm{H}), 3.69$ (t, $J=8.5 \mathrm{~Hz}$, $1 \mathrm{H}), 3.60(\mathrm{t}, J=8.0 \mathrm{~Hz}, 1 \mathrm{H}), 3.10(\mathrm{t}, J=8.0 \mathrm{~Hz}, 1 \mathrm{H}), 2.59(\mathrm{dt}, J=$ 10.0, $7.5 \mathrm{~Hz}, 1 \mathrm{H}), 2.44-2.41$ (m, 2H), 2.05 (ddd, $J=16.0,12.5,3.0 \mathrm{~Hz}$, 1H), $1.99-1.87$ (m, 2H), 1.69 (br d, $J=11.5 \mathrm{~Hz}, 1 \mathrm{H}$ ), 1.54 (dt, $J=16.0$, $4.5 \mathrm{~Hz}, 1 \mathrm{H}), 1.44$ (br d, $J=14.5 \mathrm{~Hz}, 1 \mathrm{H}), 1.19$ (qd, $J=13.0,4.5 \mathrm{~Hz}$, 1H), 1.12-1.03 (m, 2H). ${ }^{13} \mathbf{C}$ NMR (100 MHz, $\left.\mathrm{C}_{6} \mathrm{D}_{6}\right)$ : $\square$ 210.4, 74.7,

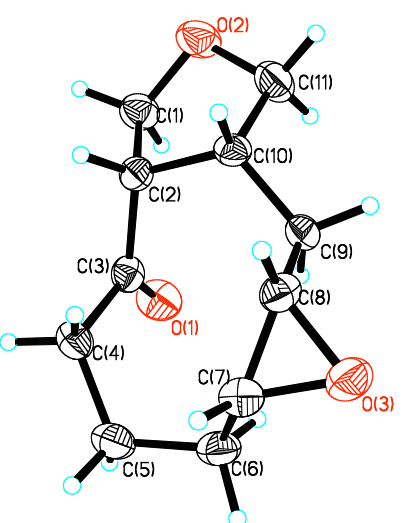


69.9, 56.6, 56.2, 53.9, 40.7, 40.3, 26.7, 23.9, 20.0. Anal. Calcd. for $\mathrm{C}_{11} \mathrm{H}_{16} \mathrm{O}_{3}$ : C, 67.32; H, 8.22. Found: C, $67.42 ; \mathrm{H}, 8.34$.

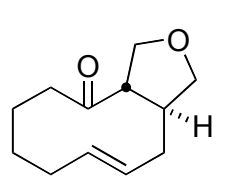

19

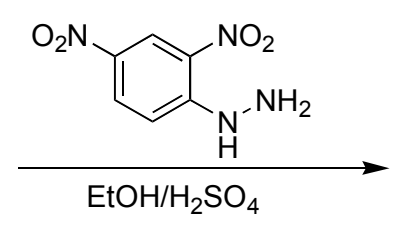<smiles>[I-]</smiles>
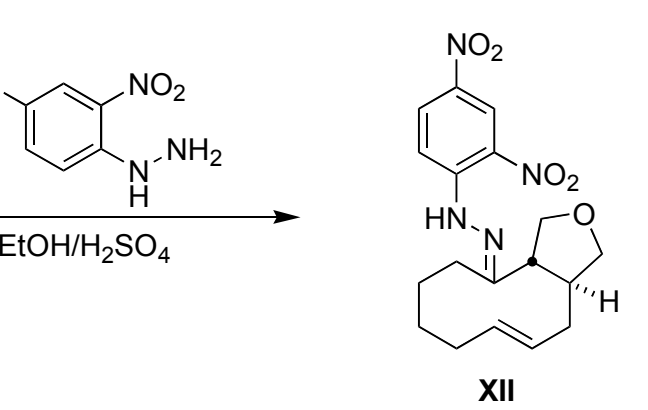

A solution of dinitrophenylhydrazine $(51.0 \mathrm{mg}, 0.257 \mathrm{mmol})$ in ethanol $(3 \mathrm{~mL})$ and $95 \%$ sulfuric acid $(0.7$ $\mathrm{mL})$ was made, and a solution of $19(22.0 \mathrm{mg}, 0.113 \mathrm{mmol})$ in ethanol $(1 \mathrm{~mL})$ was added. After stirring for $48 \mathrm{~h}$, a small amount of precipitate could be seen. The precipitate was collected by filtration, washing with cold methanol. The residue was recrystallized from $\mathrm{CH}_{2} \mathrm{Cl}_{2} /$ methanol to give dinitrophenylhydrazone XII (7.0 mg, $0.019 \mathrm{mmol}, 17 \%$ yield) as clear, orange cubes.

Compound XII: FTIR ( $\mathrm{NaCl}$, thin film): 3322 (w), $2929(\mathrm{~m}), 2856$ (m), 1620 (s), 1591 (m), 1517 (m), 1333 (s), 1310 (m), 1267 (w), 1093 (w) cm ${ }^{-1} .{ }^{1}$ H NMR (500 MHz, $\left.\mathrm{CDCl}_{3}\right)$ : $\square 11.05(\mathrm{~s}, 1 \mathrm{H}), 9.14$ (d, $J=2.5 \mathrm{~Hz}$, $1 \mathrm{H}), 8.33(\mathrm{dd}, J=10.0,2.5 \mathrm{~Hz}, 1 \mathrm{H}), 7.95$ (d, $J=10.0$ $\mathrm{Hz}, 1 \mathrm{H}), 5.61$ (ddd, $J=15.0,10.0,4.5 \mathrm{~Hz}, 1 \mathrm{H}), 5.51$ (ddd, $J=15.0,10.5,4.0 \mathrm{~Hz}, 1 \mathrm{H}), 4.21(\mathrm{t}, J=8.0 \mathrm{~Hz}$, $1 \mathrm{H}), 4.18$ (t, $J=7.5 \mathrm{~Hz}, 1 \mathrm{H}), 3.77$ (t, $J=8.5 \mathrm{~Hz}, 1 \mathrm{H})$,

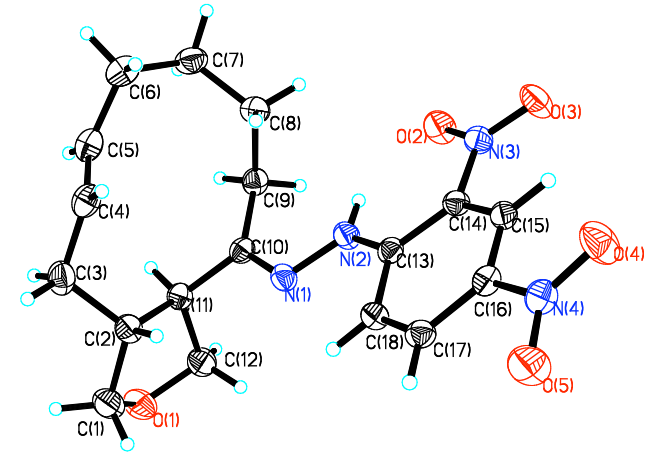
$3.56(\mathrm{t}, J=8.5 \mathrm{~Hz}, 1 \mathrm{H}), 2.96-2.83(\mathrm{~m}, 2 \mathrm{H}), 2.66(\mathrm{q}, J=$ $9.0 \mathrm{~Hz}, 1 \mathrm{H}), 2.50$ (br d, $J=12.5 \mathrm{~Hz}, 1 \mathrm{H}), 2.42-2.36(\mathrm{~m}, 2 \mathrm{H}), 2.14$ (dt, $J=15.0,4.0 \mathrm{~Hz}, 1 \mathrm{H}), 1.95-1.88(\mathrm{~m}$, 2H), $1.56(\mathrm{~s}, 1 \mathrm{H}), 1.42-1.40(\mathrm{~m}, 2 \mathrm{H}) .{ }^{13} \mathbf{C} \mathbf{N M R}\left(125 \mathrm{MHz}, \mathrm{CDCl}_{3}\right): \square 163.5,145.2,138.1,131.9,131.5$, 130.2, 129.4, 123.8, 116.7, 75.6, 74.4, 56.6, 50.7, 37.5, 36.4, 34.3, 29.9, 23.7. Anal. Calcd. for $\mathrm{C}_{18} \mathrm{H}_{22} \mathrm{~N}_{4} \mathrm{O}_{5}$ : C, 57.75; H, 5.92; N, 14.96. Found: C, 57.75; H, 5.95; N, 14.96.

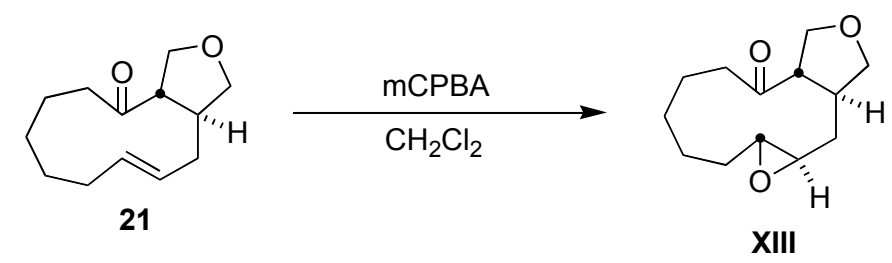

To a solution of mCPBA (46.0 mg, $0.266 \mathrm{mmol})$ in $\mathrm{CH}_{2} \mathrm{Cl}_{2}$ was added 21 (24.0 mg, $\left.0.115 \mathrm{mmol}\right)$ by syringe. After $16 \mathrm{~h}$ at room temperature, the reaction was diluted with $\mathrm{CHCl}_{3}(30 \mathrm{~mL})$ and washed with saturated aqueous $\mathrm{NaHCO}_{3}(2 \times 20 \mathrm{~mL})$. The organic layer was dried with $\mathrm{MgSO}_{4}$, concentrated in vacuo, 
and the remaining solid purified by chromatography on silica gel $(100 \%$ dichloromethane to 9:1 dichloromethane:ether) to yield epoxide XIII (20.5 mg, $0.0714 \mathrm{mmol}, 79 \%$ yield, single diastereomer) as white needles. X-ray quality crystals were grown by recrystallizing from hexanes/ether.

Compound XIII: FTIR (NaCl, thin film): 2968 (s), 2934 (s), 2872 (s), 1695 (s), 1464 (m), 1444 (w), 1089 (m), 998 (w), $952(\mathrm{~m}) \mathrm{cm}^{-1} .{ }^{1} \mathbf{H}$ NMR (400 MHz, $\left.\mathrm{C}_{6} \mathrm{D}_{6}\right)$ : $\square 3.87$ (t, $J=8.0 \mathrm{~Hz}, 1 \mathrm{H}), 3.72(\mathrm{t}, J=8.4 \mathrm{~Hz}, 1 \mathrm{H}), 3.53(\mathrm{dd}, J=$ 8.0, $7.6 \mathrm{~Hz}, 1 \mathrm{H}), 3.07$ (t, $J=8.0 \mathrm{~Hz}, 1 \mathrm{H}), 2.62(\mathrm{dqd}, J=$ $12.4,7.6,2.4 \mathrm{~Hz}, 1 \mathrm{H}), 2.42$ (q, $J=7.6 \mathrm{~Hz}, 1 \mathrm{H}), 2.31-2.24$ (m, 2H), 2.03 (ddd, $J=13.6,10.4,3.2 \mathrm{~Hz}, 1 \mathrm{H}), 1.98-1.95$ $(\mathrm{m}, 1 \mathrm{H}), 1.87-1.77(\mathrm{~m}, 2 \mathrm{H}), 1.68-1.58(\mathrm{~m}, 1 \mathrm{H})$, $1.35-1.19(\mathrm{~m}, 2 \mathrm{H}), 1.09-0.99(\mathrm{~m}, 1 \mathrm{H}), 0.97-0.85(\mathrm{~m}$,

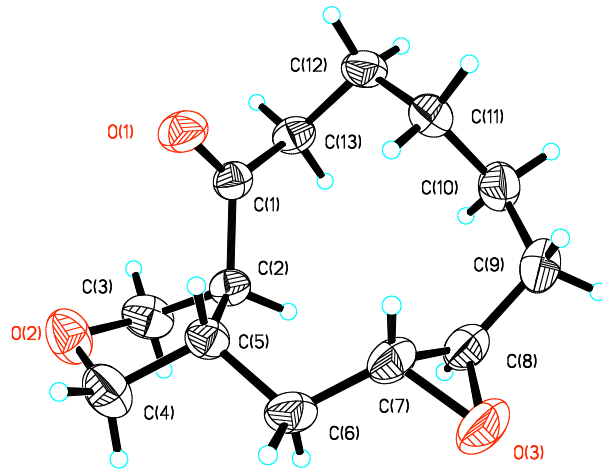
2H), 0.72 (dddd, $J=13.6,11.6,9.6,2.4 \mathrm{~Hz}, 1 \mathrm{H}$ ), 0.59 (ddd, $J=13.6,12.4,10.0 \mathrm{~Hz}, 1 \mathrm{H}) .{ }^{13} \mathbf{C}$ NMR $\left(100 \mathrm{MHz}, \mathrm{C}_{6} \mathrm{D}_{6}\right)$ : $\square 210.2,75.3,72.5,60.1,58.4,58.1,42.5$, 40.4, 36.3, 33.0, 26.9, 25.2, 23.1. Anal. Calcd. for $\mathrm{C}_{13} \mathrm{H}_{20} \mathrm{O}_{3}$ : C, 69.61; H, 8.99. Found: C, 69.51; H, 9.17.

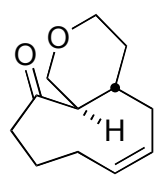

23

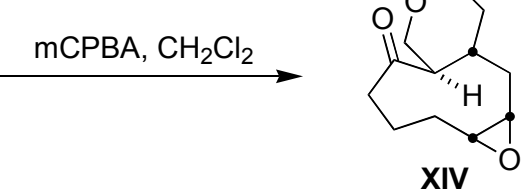

XIV

To a solution of $23(30.0 \mathrm{mg}, 0.154 \mathrm{mmol})$ in $\mathrm{CH}_{2} \mathrm{Cl}_{2}(1 \mathrm{~mL})$ was added solid mCPBA (70.0 mg, 0.394 mmol). After stirring for $16 \mathrm{~h}$, the reaction was diluted with $\mathrm{CHCl}_{3}(20 \mathrm{~mL})$ and washed with saturated $\mathrm{NaHCO}_{3}(10 \mathrm{~mL})$ and $10 \% \mathrm{KOH}(10 \mathrm{~mL})$. The organic layer was dried with $\mathrm{MgSO}_{4}$, concentrated in vacuo, and the remaining solid purified by chromatography on silica gel (3:2 hexanes:ether) to give epoxide XIV (16.0 mg, $0.0761 \mathrm{mmol}, 49 \%)$ as white needles. Crystals for X-ray diffraction were grown from pentane. (Ortep shows two molecules in unit cell)

Compound XIV: FTIR ( $\mathrm{NaCl}$, thin film): 2935 (s), 2851 (s), 1701 (s), 1457 (m), 1385 (w), 1145 (m), $1124(\mathrm{~m}), 1000(\mathrm{~m}), 860(\mathrm{~m}), 827(\mathrm{w}), 750$ (w) $\mathrm{cm}^{-1} .{ }^{1} \mathbf{H}$ NMR (500 MHz, $\mathrm{C}_{6} \mathrm{D}_{6}$ ): $\square 3.74-3.70$ (m, 2H), 3.14 (t, $J=11 \mathrm{~Hz}, 1 \mathrm{H}), 3.05(\mathrm{td}, J=11.5$, $2.5 \mathrm{~Hz}, 1 \mathrm{H}), 2.69-2.62(\mathrm{~m}, 2 \mathrm{H}), 2.28(\mathrm{td}, J=10.5$, $3.5 \mathrm{~Hz}, 1 \mathrm{H}), 1.92-1.80(\mathrm{~m}, 3 \mathrm{H}), 1.73-1.64(\mathrm{~m}$, $2 \mathrm{H}), 1.60(\mathrm{dd}, J=14,2.5 \mathrm{~Hz}, 1 \mathrm{H}), 1.30-1.25(\mathrm{~m}$,
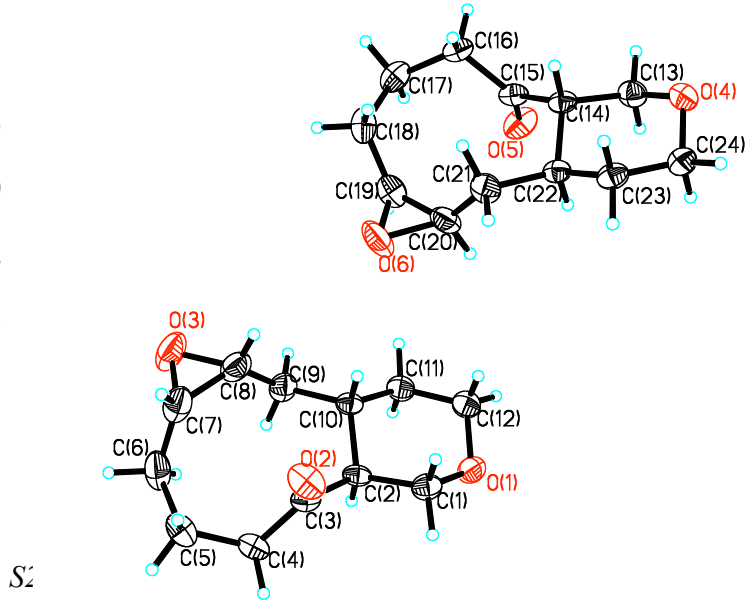
1H), 1.06 (dq, $J=12.5,4.5 \mathrm{~Hz}, 1 \mathrm{H}), 0.94-0.86(\mathrm{~m}, 3 \mathrm{H}) .{ }^{13} \mathbf{C}$ NMR (100 MHz, $\left.\mathrm{C}_{6} \mathrm{D}_{6}\right): \square 213.8,68.9,67.8$, 59.9, 58.6, 57.7, 43.4, 36.1, 34.7, 33.1, 27.0, 22.0. Anal. Calcd. for $\mathrm{C}_{12} \mathrm{H}_{18} \mathrm{O}_{3}$ : C, 68.54; H, 8.63. Found: $\mathrm{C}$, $68.47 ; \mathrm{H}, 8.91$.

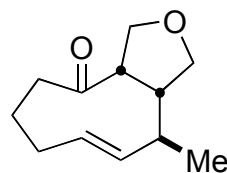

30

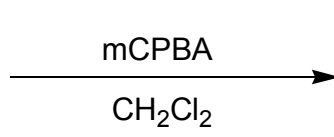

$\mathrm{CH}_{2} \mathrm{Cl}_{2}$

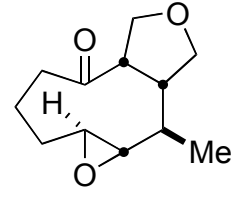

XV

To a solution of $\mathbf{3 0}(9.0 \mathrm{mg}, 0.046 \mathrm{mmol})$ in $\mathrm{CH}_{2} \mathrm{Cl}_{2}(1 \mathrm{~mL})$ was added solid mCPBA (46.0 $\mathrm{mg}, 0.266$ mmol). After stirring for $2 \mathrm{~h}$, the reaction was diluted with $\mathrm{CHCl}_{3}(15 \mathrm{~mL})$ and washed with saturated $\mathrm{NaHCO}_{3}(15 \mathrm{~mL})$. The organic layer was dried with $\mathrm{MgSO}_{4}$, concentrated in vacuo, and the remaining solid was purified by chromatography on silica gel (3:2 hexanes:ether) to give epoxide XV ( $6.4 \mathrm{mg}, 0.030$ mmol, $66 \%$ yield, single diastereomer) as white needles. Recrystallization from hexanes/ether gave crystals suitable for X-ray diffraction.

Compound XV: FTIR (NaCl, thin film): 2993 (m), 2973 (s), 2925 (s), 2868 (s), 1699 (s), 1455 (m), 1011 (m), 948 (m), 892 (s), 878 (m), 832 (m) $\mathrm{cm}^{-1} .{ }^{1} \mathbf{H}$ NMR $\left(400 \mathrm{MHz}, \mathrm{C}_{6} \mathrm{D}_{6}\right)$ : 73.99 (t, $\left.J=8.8 \mathrm{~Hz}, 1 \mathrm{H}\right), 3.86$ (t, $J=7.6 \mathrm{~Hz}, 1 \mathrm{H}), 3.84$ (t, $J=8.4 \mathrm{~Hz}, 1 \mathrm{H}), 3.30(\mathrm{t}, J=8.4 \mathrm{~Hz}, 1 \mathrm{H})$, $2.64(\mathrm{ddd}, J=10.8,9.6,7.6 \mathrm{~Hz}, 1 \mathrm{H}), 2.35(\mathrm{dt}, J=10.8,2.4 \mathrm{~Hz}, 1 \mathrm{H})$, 2.17 (dtdd, $J=14.4,12.8,4.4,1.6 \mathrm{~Hz}, 1 \mathrm{H}), 1.93$ (ddt, $J=13.6,4.8,2.8$ $\mathrm{Hz}, 1 \mathrm{H}), 1.85-1.75(\mathrm{~m}, 3 \mathrm{H}), 1.66(\mathrm{ddd}, J=16.8,6.4,2.0 \mathrm{~Hz}, 1 \mathrm{H})$, 1.23-1.07 (m, 2H), 0.79 (d, $J=6.4 \mathrm{~Hz}, 3 \mathrm{H}), 0.48$ (tdd, $J=13.8,10.8$, $5.2 \mathrm{~Hz}, 1 \mathrm{H}){ }^{13} \mathbf{C}$ NMR $\left(125 \mathrm{MHz}, \mathrm{C}_{6} \mathrm{D}_{6}\right): \square$ 209.2, 73.4, 71.2, 63.2,

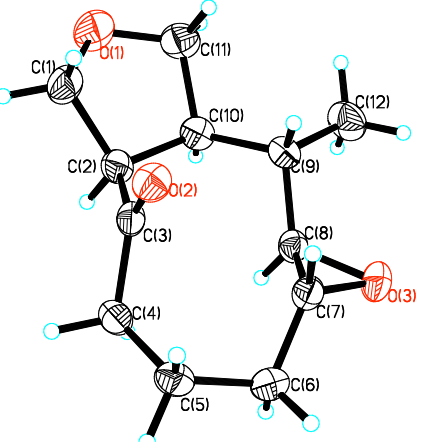
62.5, 53.6, 49.1, 43.6, 36.1, 31.6, 20.5, 16.5. Anal. Calcd. for $\mathrm{C}_{12} \mathrm{H}_{18} \mathrm{O}_{3}$ : C, 68.54; H, 8.63. Found: $\mathrm{C}$, $68.50 ; \mathrm{H}, 8.46$.

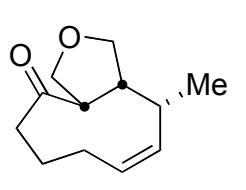

31

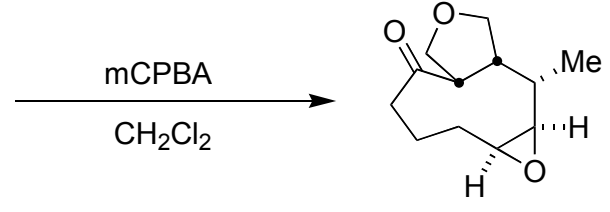

XVI

To a solution of $31(6.0 \mathrm{mg}, 0.031 \mathrm{mmol})$ in $\mathrm{CH}_{2} \mathrm{Cl}_{2}(1 \mathrm{~mL})$ was added mCPBA $(59.0 \mathrm{mg}, 0.342 \mathrm{mmol})$. The reaction was stirred for $16 \mathrm{~h}$, after which it was diluted with $\mathrm{CHCl}_{3}(15 \mathrm{~mL})$ and washed with saturated $\mathrm{NaHCO}_{3}(15 \mathrm{~mL})$. The organic layer was dried with $\mathrm{MgSO}_{4}$, concentrated in vacuo, and the remaining solid purified by chromatography on silica gel (3:2 hexanes:ether) to give epoxide XVI (5.8 mg, 0.028 
mmol, $89 \%$ yield, single diastereomer) as white needles. Recrystallization from hexanes/ether gave crystals suitable for X-ray diffraction.

Compound XVI: FTIR (NaCl, thin film): 2964 (s), 2872 (s), 1705 (s), 1460 (m), 1370 (m), 1210 (w), $1110(\mathrm{~m}), 1073(\mathrm{~m}), 959(\mathrm{~m}), 938(\mathrm{~m}), 805(\mathrm{~m}) \mathrm{cm}^{-1} .{ }^{1} \mathbf{H} \mathbf{~ N M R}\left(400 \mathrm{MHz}, \mathrm{C}_{6} \mathrm{D}_{6}\right)$ : $\square 4.25(\mathrm{dd}, J=8.8,6.4$ $\mathrm{Hz}, 1 \mathrm{H}), 3.67(\mathrm{t}, J=8.4 \mathrm{~Hz}, 1 \mathrm{H}), 3.66(\mathrm{t}, J=8.4 \mathrm{~Hz}, 1 \mathrm{H}), 3.60$ (t, $J$ $=8.8 \mathrm{~Hz}, 1 \mathrm{H}), 2.65(\mathrm{dt}, J=10.4,3.6 \mathrm{~Hz}, 1 \mathrm{H}), 2.54(\mathrm{dd}, J=10.4$, $4.0 \mathrm{~Hz}, 1 \mathrm{H}$ ), 2.49 (dt, $J=11.6,8.0 \mathrm{~Hz}, 1 \mathrm{H}$ ), 2.17 (dddd, $J=11.6$, 9.6, 7.6, $3.6 \mathrm{~Hz}, 1 \mathrm{H}), 2.01-1.98(\mathrm{~m}, 1 \mathrm{H}), 1.91(\mathrm{dq}, J=13.6,3.6$ $\mathrm{Hz}, 1 \mathrm{H}), 1.79-1.64(\mathrm{~m}, 2 \mathrm{H}), 1.56$ (ddd, $J=10.8,7.2,4.0 \mathrm{~Hz}, 1 \mathrm{H})$, $1.22-1.15(\mathrm{~m}, 1 \mathrm{H}), 0.88(\mathrm{~d}, J=6.8 \mathrm{~Hz}, 3 \mathrm{H}), 0.83-0.73(\mathrm{~m}, 1 \mathrm{H})$. ${ }^{13}$ C NMR (125 MHz, $\left.\mathrm{C}_{6} \mathrm{D}_{6}\right)$ : $\square 210.8,68.7,68.1,59.7,57.5,53.2$, 47.9, 42.9, 29.4, 29.3, 20.3, 18.5. Anal. Calcd. for $\mathrm{C}_{12} \mathrm{H}_{18} \mathrm{O}_{3}$ : C, 68.54; H, 8.63. Found: C, 68.71; H, 8.92.

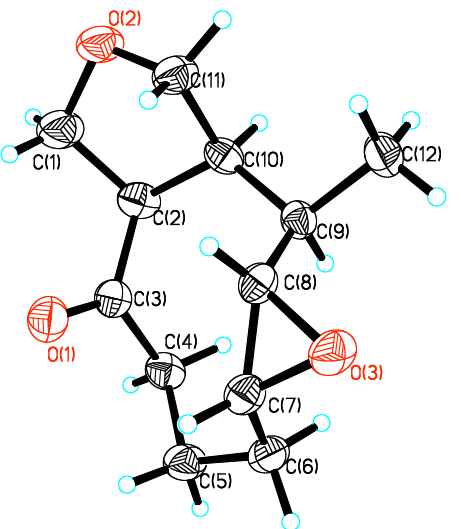

